\title{
Spike-Based Functional Connectivity in Cerebral Cortex and Hippocampus: Loss of Global Connectivity Is Coupled to Preservation of Local Connectivity During Non-REM Sleep
}

\author{
Umberto Olcese, ${ }^{\star}$ Jeroen J. Bos, ${ }^{\star}$ Martin Vinck, ${ }^{\star}$ Jan V. Lankelma, Laura B. van Mourik-Donga, Friederike Schlumm, \\ and $\odot$ Cyriel M.A. Pennartz \\ Cognitive and Systems Neuroscience Group, Swammerdam Institute for Life Sciences, University of Amsterdam, 1098XH Amsterdam, The Netherlands
}

Behavioral states are commonly considered global phenomena with homogeneous neural determinants. However, recent studies indicate that behavioral states modulate spiking activity with neuron-level specificity as a function of brain area, neuronal subtype, and preceding history. Although functional connectivity also strongly depends on behavioral state at a mesoscopic level and is globally weaker in non-REM (NREM) sleep and anesthesia than wakefulness, it is unknown how neuronal communication is modulated at the cellular level. We hypothesize that, as for neuronal activity, the influence of behavioral states on neuronal coupling strongly depends on type, location, and preceding history of involved neurons. Here, we applied nonlinear, information-theoretical measures of functional connectivity to ensemble recordings with single-cell resolution to quantify neuronal communication in the neocortex and hippocampus of rats during wakefulness and sleep. Although functional connectivity (measured in terms of coordination between firing rate fluctuations) was globally stronger in wakefulness than in NREM sleep (with distinct traits for cortical and hippocampal areas), the drop observed during NREM sleep was mainly determined by a loss of inter-areal connectivity between excitatory neurons. Conversely, local (intra-area) connectivity and long-range (inter-areal) coupling between interneurons were preserved during NREM sleep. Furthermore, neuronal networks that were either modulated or not by a behavioral task remained segregated during quiet wakefulness and NREM sleep. These results show that the drop in functional connectivity during wake-sleep transitions globally holds true at the cellular level, but confine this change mainly to long-range coupling between excitatory neurons.

Key words: brain network integration; brain states; functional connectivity; neuronal network analysis; spiking activity; wakefulness and sleep

\section{Significance Statement}

Studies performed at a mesoscopic level of analysis have shown that communication between cortical areas is disrupted in non-REM sleep and anesthesia. However, the neuronal determinants of this phenomenon are not known. Here, we applied nonlinear, information-theoretical measures of functional coupling to multi-area tetrode recordings from freely moving rats to investigate whether and how brain state modulates coordination between individual neurons. We found that the previously observed drop in functional connectivity during non-REM (NREM) sleep can be explained by a decrease in coupling between excitatory neurons located in distinct brain areas. Conversely, intra-area communication and coupling between interneurons are preserved. Our results provide significant new insights into the neuron-level mechanisms responsible for the loss of consciousness occurring in NREM sleep.

\section{Introduction}

Behavioral states are commonly considered global brain processes (Pace-Schott and Hobson, 2002) despite the fact that neural activity is highly regulated at the local level; different brain areas display distinct types of activity during both wakefulness and sleep [e.g., cortical slow waves (Steriade et al., 2001) and hippocampal sharp wave ripples (Klausberger et al., 2003)]. At the cellular level, behavioral states differentially affect neuronal 
firing activity depending on brain area under scrutiny, neuronal subtype, and preceding history (Hirase et al., 2001a; Greenberg et al., 2008; Gentet et al., 2010; Lansink et al., 2010; Vyazovskiy et al., 2011; Sachidhanandam et al., 2013; Goltstein et al., 2015). Recent studies have highlighted a strong relationship among brain state, patterns of neural activity, and behavioral performance. Specifically, brain states characterized by moderate arousal and firing activity with an intermediate level of synchrony correlate with better behavioral performance in sensory-motor tasks than during either high arousal [e.g., locomotion (McGinley et al., 2015a; Vinck et al., 2015a)] or low arousal, sleep, anesthesia, or fatigue (Vyazovskiy et al., 2011; Goltstein et al., 2015; McGinley et al., 2015a, 2015b).

Functional connectivity has also been shown to depend strongly on behavioral state at a macroscopic level (Massimini et al., 2005; Lu et al., 2012; Park and Friston, 2013). In particular, a decrease in cortical connectivity has been observed in NREM sleep and anesthesia with respect to wakefulness (Massimini et al., 2005; Ferrarelli et al., 2010; Lewis et al., 2012; Bettinardi et al., 2015). Most previous studies used techniques accessing the macroscopic and mesoscopic scales: electroencephalographic (EEG) and local field potential (LFP) recordings. Because brain activity and behavior are determined by both neuronal spiking activity and communication between neurons, we hypothesize that, similarly to firing activity, functional connectivity between neurons may also be highly regulated across behavioral states. Specifically, we hypothesize that, as for neuronal spiking activity, the influence of behavioral states on neuronal coupling may strongly depend on the anatomical location of involved neurons. Recent studies support this view because connections between different brain areas undergo specific functional changes in the transition between wakefulness and anesthesia. Cell-attached recordings showed that synaptic connections from the cingulate cortex to the primary visual cortex are not affected by brain-state transitions (Zhang et al., 2014). Conversely, other top-down pathways impinging onto the visual cortex (specifically from the retrosplenial cortex) were shown, in two-photon calcium imaging, to lose their functional role during anesthesia (Makino and Komiyama, 2015). Moreover, because distinct neuronal subtypes play different roles in transferring information between brain regions (Gentet et al., 2010; Olcese et al., 2013; Makino and Komiyama, 2015), we hypothesize a differential brain-statedependent modulation of coupling between excitatory and inhibitory neurons. We expect coupling between principal (excitatory) cells to be weaker than that between interneurons in brain states with low levels of information processing (e.g., NREM sleep). Conversely, interneurons have been shown to maintain a role in regulating cortical activity during NREM sleep, namely by modulating how neighboring pyramidal neurons respond to thalamic inputs (Contreras et al., 1997). Furthermore, we hypothesize that functionally integrated neuronal networks, for example, sets of neurons involved in performing a specific task, will remain strongly interconnected during resting states as

\footnotetext{
University of Amsterdam, in particular Gerrit Hardeman, Eric Hennes, Harry Beukers, Ron Manuputy, Matthijs Bakker, and Ed de Water.

The authors declare no competing financial interests.

*U.O., J.J.B., and M.V. contributed equally to this work.

Correspondence should be addressed to Cyriel M.A. Pennartz, Cognitive and Systems Neuroscience Group,

Swammerdam Institute for Life Sciences, University of Amsterdam, Science Park 904, 1098XH Amsterdam, The Netherlands. E-mail: c.m.a.pennartz@uva.nl.

DOI:10.1523/JNEUROSCI.4201-15.2016

Copyright $\odot 2016$ the authors $\quad 0270-6474 / 16 / 367677-17 \$ 15.00 / 0$
}

a consequence of long-term plastic changes in the synaptic connections between the neurons involved.

By applying nonlinear measures of functional coupling to multi-area tetrode recordings in freely moving rats (Vinck et al., 2015b), we found that functional coupling between individual neurons, measured in terms of coordinated firing rate fluctuations (Harris and Thiele, 2011), not only changes as a function of brain state (and is generally stronger in wakefulness than NREM sleep), but is also highly dependent on distinct brain areas (neocortex vs hippocampus), distance between neurons (within and between brain regions), neuronal subtypes (excitatory vs inhibitory cells), and on functional properties of individual neurons. Our results indicate that behavioral states are global brain phenomena with neural correlates that are highly localized processes.

\section{Materials and Methods Subjects}

All animal experiments were conducted according to the National Guidelines on Animal Experiments and were approved by the Animal Experimentation Committee of the University of Amsterdam. Data were collected from three male Lister hooded rats. Animals were kept under a reversed day/night cycle (lights off: 8:00 A.M., lights on: 8:00 P.M.). During both training and experimental (recording) sessions, animals were food restricted to maintain their body weight at $85 \%$ of ad libitum fed animals. Rats had ad libitum access to water during all phases of the experiment.

\section{Behavioral setup}

Animals were trained to perform a two-choice visual discrimination task on a figure-eight maze (see gray tracks in Fig. 8A). Each trial started with the animal confined to the middle arm of the maze by two movable Plexiglas blocking walls. Animals were trained to attend to visual stimuli presented simultaneously on two monitors (LCD, 15 inch, Dell; see blue lines in Fig. 8A). The stimuli consisted of inverted equiluminant Wingdings (Microsoft) figures (either a diamond or an airplane, randomized across animals) that had the same proportions of black and white pixels. Stimuli started when animals interrupted an infrared beam located in front of the movable Plexiglas door that was closest to the monitors (see black square in Fig. 8A). After $4.2 \mathrm{~s}$, the front wall was removed. Animals were trained to choose the side arm of the maze (either left or right) corresponding to the screen where the positive conditioned stimulus was shown (airplane or diamond shape). If animals chose the correct side of the maze, they would be rewarded with two or three pellets (dustless precision pellets, $14 \mathrm{mg}$; BioServe) placed in a ceramic cup (see black circles in Fig. 8A). Upon choosing one side of the maze, animals would encounter strips of sandpaper on the walls of the maze (see orange areas in Fig. 8A). Visual stimuli were terminated when animals crossed an infrared beam located at the end of the area where sandpaper was present. The grain of the sandpaper predicted the amount of reward that the animals would receive after choosing the correct side arm: two pellets for fine sandpaper (P40) and three pellets for coarse sandpaper (P180). One pellet was also provided in the middle arm (see black square in Fig. $8 \mathrm{~A}$ ) if animals had chosen the correct side arm in the previous trial. Video acquisition (see Fig. $1 E$ ) was synchronized to electrophysiological recordings. Eight infrared beams were used to synchronize animal position to electrophysiological recordings and to control the behavioral setup via a custom-made MATLAB program (The MathWorks). For the purposes of the current study, we were only interested in discriminating whether the firing rate of neurons was significantly modulated during certain phases of the task (see "Data analysis" section), so we did not discriminate between correct and incorrect trials. All animals were highly trained on the task and performed $60.1 \pm 18.7$ (mean \pm SD) trials per recording session, with an average success rate of $59.2 \pm 0.9 \%$ (mean \pm SE), which was significantly different from chance $\left(p=2.1 \times 10^{-8}\right)$. The success rate had reached a stable value for all animals at the end of the training period. Animals were trained for $\sim 1 \mathrm{~h} 5 \mathrm{~d}$ /week. 


\section{Surgical procedure and recording drive}

The right brain hemisphere of each rat was implanted with a custombuilt tetrode microdrive, each containing 36 individually movable tetrodes. Eight recording tetrodes were directed to the monocular portion of the primary visual cortex $(\mathrm{V} 1 \mathrm{M},-6.0 \mathrm{~mm}$ posterior and $-3.2 \mathrm{~mm}$ lateral to bregma), eight to the hippocampus CA1 field (HPC, $-3.5 \mathrm{~mm}$ posterior and $-2.4 \mathrm{~mm}$ lateral), eight to the barrel field of the primary somatosensory cortex ( $\mathrm{S} 1 \mathrm{BF},-3.1 \mathrm{~mm}$ posterior and $-5.1 \mathrm{~mm}$ lateral), and eight to the perirhinal cortex $(\mathrm{PRH},-5 \mathrm{~mm}$ posterior and $-5 \mathrm{~mm}$ lateral, with an angle of $17^{\circ}$ relative to the skull midline). One additional tetrode per area was used as a local reference. Approximately 20-30 min before surgery, each animal received a subcutaneous injection of buprenorphin (Buprecare, 0.01-0.05 mg/kg), meloxicam (Metacam, $2 \mathrm{mg} / \mathrm{kg}$ ), and Baytril (5 mg/kg). Animals were kept under isoflurane anesthesia (1-3\%); body temperature was maintained between $35^{\circ} \mathrm{C}$ and $36^{\circ} \mathrm{C}$ via a heating pad. Six surgical screws were implanted into the skull to fixate the microdrive to the skull using dental cement; the left parietal screw was used as the signal ground. Four craniotomies (each $\sim 1.8 \mathrm{~mm}$ in diameter) were made, corresponding to the above-mentioned coordinates to enable access to the brain areas of interest. After removing the dura, the tetrodes of the four bundles were lowered $0.4-1.0 \mathrm{~mm}$ (depending on the target area) into the cortex. Animals were allowed to recover for a week, with ad libitum food and water available. Tetrodes were gradually lowered to their target region over the course of the first 7-9 d after implantation. Tetrode position in relation to the target region was estimated based on the total number of turns of the guide screws (Lansink et al., 2007; Vinck et al., 2015b) and was also based on online monitoring of the LFP and spiking signals. Finally, tetrode positions were confirmed via histology.

\section{Histology}

After the final recording session, a small electrolytic lesion $(12 \mu \mathrm{A}$ for $10 \mathrm{~s}$ ) was made on one lead per tetrode to mark the endpoint of the tetrode. Animals were deeply anesthetized with Nembutal (sodium pentobarbital, $60 \mathrm{mg} / \mathrm{ml}, 1.0 \mathrm{ml}$, i.p.; Ceva Sante Animale) and transcardially perfused with a $0.9 \% \mathrm{NaCl}$ solution, followed by a $4 \%$ paraformaldehyde solution ( $\mathrm{pH} 7.4$, phosphate buffered). Coronal sections with a thickness of $40 \mu \mathrm{m}$ were obtained with a vibratome. Sections were then stained with cresyl violet to reconstruct tetrode tracks and localize their endpoints.

\section{Data acquisition and spike sorting}

Thirty-two tetrodes (nichrome; $14 \mu \mathrm{m}$ diameter per lead, gold-plated to $500-800 \mathrm{k} \Omega$ impedance at $1 \mathrm{kHz}$; California Fine Wire) were used to record neural activity with a 128-channel Digital Cheetah system (Neuralynx). Signals were routed through a unity-gain preamplifier headstage and a 128-channel, automated commutator (Neuralynx) and were digitally band-pass filtered between 600 and $6000 \mathrm{~Hz}$ for spike recordings. Whenever the signal on any of the leads of a tetrode crossed a preset voltage threshold, $1 \mathrm{~ms}$ of activity from all 4 tetrode leads was digitized at $32 \mathrm{kHz}$, corresponding approximately to a whole action potential waveform. LFPs were recorded from all tetrodes, continuously sampled at 2 $\mathrm{kHz}$, and band-pass filtered between 1 and $500 \mathrm{~Hz}$. Action potentials were assigned to single neurons by using a semiautomated spike sorting algorithm (KlustaKwik, Ken Harris and MClust 3.5, A.D. Redish). Individual spike clusters were considered as single neurons when no more than $0.1 \%$ of all interspike intervals were shorter than $2 \mathrm{~ms}$. During recordings, the video acquisition of the whole setup was performed at 25 Hz. Interruptions of infrared beams were monitored at $32 \mathrm{kHz}$.

Each recording session consisted of three phases. The middle phase lasted $\sim 1.5 \mathrm{~h}$, during which animals performed the behavioral task in the dark (red lights only; see Fig. $1 E$, right). Before and after this phase, animals were placed in a flower pot on top of the maze (Fig. 1E, left) and usually remained quiet or asleep for $30 \mathrm{~min}$ to $1 \mathrm{~h}$ under dim lights. The two resting phases were pooled together in the present study. No significant difference in any information theoretical measure was observed between them $(p>0.05)$. For each animal, we selected two recording sessions during which we found at least four neurons in each recorded region and at least $30 \mathrm{~min}$ spent into each state: active wakefulness (AW), quiet wakefulness (QW) and NREM. Tetrodes were always moved between recordings sessions.

Data were recorded during a total of six recording sessions in three different rats. On average, we recorded $13 \pm 4$ neurons from S1BF, $6 \pm 2$ from V1M, $24 \pm 10$ from PRH, and $22 \pm 4$ from HPC. Behavioral scoring resulted, on average, in $6293 \pm 543 \mathrm{~s}$ of AW per session, $2277 \pm 169 \mathrm{~s}$ of QW, and $1774 \pm 390 \mathrm{~s}$ of NREM. All numbers are average \pm SE.

\section{Data analysis: preprocessing}

Data analysis was performed by custom-made scripts in MATLAB (The MathWorks).

\section{Motion tracking}

Motion tracking was performed by a semiautomated custom implementation of object tracking via a partial least-squares method (Wang et al., 2012), in which the object to be tracked was the headstage on top of the rat's head (see Fig. 1E). Each headstage was equipped with four LEDs to facilitate its detection in the dark.

\section{Behavioral scoring}

Behavioral scoring was performed manually, following standard methodologies applied to rodent behavior based on cortical EEG/LFPs and motor activity (Franken et al., 1991, 2001; Vyazovskiy et al., 2009). Because rodent sleep is known to be highly fragmented, we divided the recording sessions into $4 \mathrm{~s}$ epochs and scored each epoch individually. Scoring was performed by evaluating, simultaneously, several cortical LFP channels, the LFP power spectrum in each epoch, motor activity (defined as the frame-by-frame displacement of the rat's head, as identified by our motion tracking algorithm), and raw video files. The behavioral state of recorded animals was scored into the different behavioral states: AW, QW, and NREM sleep. Any epoch that did not fulfill the criteria for AW, QW, or NREM was discarded. For all subsequent analyses, only sufficiently long periods of AW, QW, or NREM (defined as periods lasting at least $30 \mathrm{~s}$ ) were taken into account. Because only a limited amount of REM sleep was present in our dataset, REM sleep epochs were not taken into account for further analysis. AW was characterized by the presence of cortical theta rhythm (between 6 and $10 \mathrm{~Hz}$; see Fig. $1 B$ ), by low levels of neocortical slow wave activity (SWA, defined as the total power between 0.5 and $4 \mathrm{~Hz}$; Vyazovskiy et al., 2009), and by detectable levels of motor activity. Cortical LFP traces showed a typical pattern with low-amplitude, high-frequency oscillations. QW had the same electrophysiological correlates of AW, but was characterized by the absence of detectable motion (except nonexploratory movements such as grooming that were not reliably captured by our motion-tracking algorithm and were thus included into QW). Periods during which animals were not moving, but were waiting for or observing visual stimuli or consuming reward pellets, were included into AW. AW was mostly limited to periods when animals were performing the task. QW was mostly limited to periods when animals were resting in the flower pot. Following the conventions used in the analysis of NREM sleep in rodents, this state was mainly characterized using the neocortical features typical of slowwave sleep and no further subdivision in stages was performed (Franken et al., 2001; Vyazovskiy et al., 2009, 2011; Bastianini et al., 2015). NREM epochs were thus characterized by high SWA in neocortex and by weak motor activity and were limited to the periods during which animals were resting in the flower pot. Examples of cortical LFP traces for periods that were scored as AW, QW, or NREM are shown in Figure 1A. Example power spectra from epochs scored as AW, QW, and NREM are shown in Figure $1 B$. We validated the results of our behavioral scoring procedure a posteriori, by plotting, for each recording session separately, several electrophysiological and behavioral parameters. SWA was slightly increased in QW epochs compared with AW epochs, but was dramatically larger in NREM epochs (Fig. 1C). Although we did not use hippocampal traces to perform the scoring of states, it is known that hippocampal sharp wave ripples are a typical feature of QW and NREM, but occur less frequently in AW (Ylinen et al., 1995; Kudrimoti et al., 1999; Pennartz et al., 2004; Girardeau and Zugaro, 2011). We therefore measured hippocampal ripples as an additional way to evaluate the effectiveness of our behavioral 
scoring procedure. Hippocampal ripples were indeed scarce in AW epochs, but very frequent in QW and NREM epochs (Fig. 1D). Finally, we summarized the results of our motion-tracking algorithm (Fig. $1 E$ ) by computing the average pixel displacement per epoch (Fig. $1 F$ ). Average motion was larger in AW than in both QW and NREM.

\section{Determination of putative excitatory and inhibitory neurons}

Neurons were subdivided into putative excitatory neurons and putative pyramidal neurons on the basis of their action potential waveforms (Barthó et al., 2004; Iurilli et al., 2013). In particular, broad action potentials have been associated with excitatory, regular-firing neurons, and narrow action potentials with fast-spiking interneurons, in particular with parvalbumin-positive interneurons (Iurilli et al., 2013). For each isolated neuron, we computed an average action potential waveform. We found that the parameter that was well able to divide the action potentials we recorded into two distinct subpopulations was the peak-to-trough delay. Neurons with a peak-to-trough delay shorter than $0.25 \mathrm{~ms}$ were classified as putative inhibitory neurons (Iurilli et al., 2013); all others as putative excitatory neurons (see Fig. 6A). Of 381 recorded neurons, 66 were classified as putative inhibitory neurons. This corresponds to $17.3 \%$ of all recorded neurons and is close to the presumed proportion of interneurons in both the neocortex (Harris and Shepherd, 2015) and hippocampal region CA1 (Wierenga et al., 2010).

\section{Determination of task-modulated and non-modulated neurons}

Peri-event time histograms (PETHs) were constructed for individual neurons based on the time each of the eight infrared beams present on the maze was crossed by animals during task performance (see examples in Fig. $8 B$ ). PETHs were constructed in the $[-1010] \mathrm{s}$ range with a time bin of $250 \mathrm{~ms}$. Because recorded neurons could respond to a variety of events (e.g., sensory stimuli, position on the maze), we visually identified for each region and infrared beam a baseline and response window (baseline window: $[-10-8] \mathrm{s}$ and $[810] \mathrm{s}$ range around the crossing of a beam; response window: [-2 2] s range around the crossing of a beam; see Fig. $8 B$ ). Neurons were classified as task-modulated (TM) if their firing rates in the response window were significantly higher or lower than the average firing rate in the baseline window (Iurilli et al., 2013; Olcese et al., 2013) for at least one of the eight PETHs (because we computed one PETH per infrared beam crossing). Of 381 recorded neurons, 192 were classified as responsive. This corresponds to $50.1 \%$ of all recorded neurons.

\section{Detection of hippocampal ripples}

An automated procedure was implemented to detect ripples in the hippocampal LFP signals. LFP signals were band-pass filtered in the $100-$ $200 \mathrm{~Hz}$ range and smoothed with a 30-point Gaussian window. Ripples were identified as portions of the filtered trace exceeding a threshold, defined for each signal as 3.5 times the SD of the whole trace. Each ripple event had to have a minimal duration of $6 \mathrm{~ms}$. Events closer than $100 \mathrm{~ms}$ were grouped together.

\section{Data analysis: information-theoretical measures}

To investigate functional connectivity in our dataset, we used various information-theoretical measures. Because of their nonlinear nature, these measures are more general than their linear equivalents (Steuer et al., 2002) (e.g., correlation, Granger causality) and are therefore highly suitable to be used for neuronal data.

All information-theoretical measures that we used were based on firing rates of single neurons. Firing rates were computed by binning spike times into non-overlapping bins. The width of temporal bins was varied between $50 \mathrm{~ms}$ and $1 \mathrm{~s}$ in steps of $50 \mathrm{~ms}$. The firing rate was subdivided into amplitude bins. The number of amplitude bins was varied between 2 and 50. For each neuron, amplitude bins were constructed by dividing the range between the lowest and highest recorded firing rate of each neuron into $n$ linearly equispaced, non-overlapping bins. For each information-theoretical measure, a subset of time and amplitude bins was selected [see the following sections on conditional delayed automutual information (cDAMI), conditional mutual information (cMI), and the Results section for details). Although several methods have been proposed to select the size of time bins, we chose an empirical approach similar to one described previously (Reinagel and Reid, 2000). Importantly, whereas shorter time scales (i.e., shorter than $50 \mathrm{~ms}$ ) are usually reported when computing information-theoretical measures on spike trains (Arabzadeh et al., 2004; Gourévitch and Eggermont, 2007), our data mostly consisted of spontaneous activity, during which relatively low firing rates (mostly $<10 \mathrm{~Hz}$; see Fig. $6 \mathrm{~B}, C$ ) preclude the use of short time bins. Indeed, we were not able to find any clear relationship between cMI computed over a short time scale (2-50 ms) and cross-correlograms between individual neurons (data not shown). By not focusing on a single time bin and number of amplitude bins, we aimed to make our estimates more robust. Because values within the bin range were normally distributed for both cMI and cDAMI, we computed the average cMI or CDAMI value over the selected range of time and amplitude bins. As an indication of the robustness of our methods, we verified that the results obtained with equispaced binning were fully compatible with those obtained using equipopulated binning (temporal bins: 600-900 ms, amplitude bins: $5-15 ; p>0.05$ for each brain state between cMI values computed with the two different binning procedures, Wilcoxon paired signed-rank test; see also Fig. 2C,D; cf. Magri et al., 2009).

To de-bias the information-theoretical measures (which is necessary to take into account finite sampling and the different number of epochs for distinct behavioral states), we applied a different shuffling procedure for each measure (Wallisch et al., 2008), which consisted of randomizing the order of one (or more) sequences of firing rates for individual neurons (see sections on cDAMI and cMI for details). Each shuffling was repeated 10 times and the average result was subtracted from the value computed on the actual data to obtain an unbiased value. cMI values were considered to be significantly larger than 0 if their non-unbiased value exceeded the bootstrap-estimated (100 repetitions) $95^{\text {th }}$ percentile of the distribution of shuffled values (Hatsopoulos et al., 1998). We verified the effectiveness of this debiasing procedure, which previous studies indicated to be less effective than more complex ones (Panzeri et al., 2007), by computing cMI values on a randomly selected subportion of the dataset (for each recording session and brain state, we randomly retained $50 \%$ of all epochs). Because the results that we obtained did not show major changes (the only significant, yet minor, difference was observed when pooling together all pairs of neurons during NREM, $p=$ 0.03 , Wilcoxon signed-rank test; no other significant difference was observed when subdividing neurons into brain areas or functionally defined subtypes), we conclude that the de-biasing procedure that we used effectively corrected bias due to dataset size effects.

We also explored the possibility of computing cMI values separately for up and down states occurring during NREM by implementing a procedure to discriminate up and down states based on the phase of LFP signals (Saleem et al., 2010). However, the resulting cMI values computed separately for up and down states appeared not to be reliable (and not different from zero), possibly due to the lack of a sufficient amount of data to properly estimate cMI. For this reason, we did not discriminate between up and down states in the current study.

\section{$c M I$}

cMI (Steuer et al., 2002; MacKay, 2003) was computed between pairs of neurons via the following formula:

$$
c M I(X, Y \mid h)=\sum_{x \in X, y \in Y} p(x, y \mid h) \log \frac{p(x, y \mid h)}{p(x \mid h) p(y \mid h)}
$$

Where $X$ and $Y$ denote the set of all amplitude bins for two distinct neurons, $x$ is one amplitude bin within $X, y$ is one amplitude bin within $Y, h$ is the behavioral state during which firing rate values have been considered, and $\log$ is the logarithm in base 2. Shuffled estimates were computed by independently scrambling the order of firing rates for neuron $X$ and $Y$, thus affecting the joint probability distribution $p(x, y \mid h)$, but not marginal ones $p(x \mid h)$ and $p(y \mid h)$. We computed cMI for an ample range of time bin width $(50-1000 \mathrm{~ms}$ in steps of $50 \mathrm{~ms})$ and number of amplitude bins (2-50) and then selected a smaller range for further analysis. cMI was averaged across time bins ranging from 600 to $900 \mathrm{~ms}$ and the number of amplitude bins ranging from 10 to 20 (see black rectangle in Figs. $2 C, 4 A$ ). 


\section{Relationship between $c M I$ and firing rates}

To investigate whether cMI was monotonically related to the average firing rates of neurons $X$ and $Y$, we used a linear mixed-effects model to analyze the relationship between cMI values and the average firing rates of neurons $X$ and $Y$ in the different behavioral states. Although the relationship between $\mathrm{cMI}$ and the average firing rate of either neuron $X$ or $Y$ was significant $(p=0.025)$, this was by several orders of magnitude less significant than the relationship between $\mathrm{cMI}$ and the interaction term between the average firing rates of neurons $X$ and $Y\left(p=2.5 \times 10^{-14}\right)$. This confirmed that cMI is related to the interaction between the firing rates of different neurons rather than to their individual values.

\section{Relationship between cMI and task-related modulation of neuronal activity}

Finally, a possibly confounding factor in our analyses is that cMI values might reflect not only a different level of coupling based on the specific behavioral state but also (in particular during AW) correlations between the activity of different neurons due to coordinated neuronal responses to sensorimotor inputs (e.g., during the behavioral task). This appears unlikely for two reasons:

First, cMI values during AW are comparable or lower than those during QW (see Figs. 2, 3, 4). This excludes that cMI values during AW are mostly driven by external or motor variables (e.g., sensory stimuli or locomotion), which would increase spurious (i.e., externally driven) correlations between the activity of distinct neurons. If this would have been the case, cMI values during AW would have been higher than during QW.

Second, we performed an additional analysis to verify that cMI values during AW were not simply driven by modulation of neuronal activity during the behavioral task. Locomotion and quiescence are known to greatly affect both cortical and hippocampal activity patterns (McNaughton et al., 1983; McGinley et al., 2015a). In particular during locomotion, neurons in the hippocampus show higher theta LFP power than in quiescence, theta phase precession, and speed-dependent firing rates (McNaughton et al., 1983; Fuhrmann et al., 2015). If cMI were primarily driven by sensory and motor variables, one would expect cMI within the hippocampus and between cortical areas and the hippocampus to be higher during locomotion than during quiescence. However, we found this not to be the case. AW epochs were subdivided into periods with or without locomotion, that is, when animals were either moving along the maze or waiting for stimulus presentation in the middle arm. cMI during AW was not significantly different between periods of locomotion or quiescence for the intrahippocampal and cortico-hippocampal pairs of neurons ( $p>0.05$ for HPC-HPC, PRIM-HPC, and PRH-HPC pairs, paired Wilcoxon signed-rank test, PRIM is the combination of V1M and $\mathrm{S} 1 \mathrm{BF}$ neurons). Crucially, this was the case for connections between pairs of TM neurons as well as pairs of non-TM neurons. Restricting the analysis to homogeneous phases of the task (i.e., specific sensory stimuli or narrow portions of the maze) is expected to lead to correlated firing activity, and thus high cMI values, between neurons. Instead, computing functional coupling over broad, heterogeneous epochs, as we did here, makes cMI values dependent on the overall behavioral state rather than of specific external variables.

In conclusion, cMI values as computed here reflect interactions (i.e., coupling) between neurons and are unlikely to result from spurious correlations due to external inputs or motor activity.

\section{cDAMI}

cDAMI (Pompe et al., 1998; MacKay, 2003) was computed by the following equation:

$$
\begin{aligned}
\operatorname{cDAMI}(X, d \mid h)=\operatorname{MI}\left(X, X^{(\mathrm{d})} \mid h\right) & \\
& =\sum_{x \in X} p\left(x, x^{(\mathrm{d})} \mid h\right) \log \frac{p\left(x, x^{(\mathrm{d})} \mid h\right)}{p(x \mid h) p\left(x^{(\mathrm{d})} \mid h\right)}
\end{aligned}
$$

Where $X$ is the set of all amplitude bins for the firing rate of one specific neuron; $x$ is one amplitude bin within $X, X^{(\mathrm{d})}$ and $x^{(\mathrm{d})}$ indicate the firing rates for the same neuron delayed by $d$ time bins, $X^{(\mathrm{d})}$ is the set of all delayed firing rate bins, $x^{(\mathrm{d})}$ an individual bin within $X^{(\mathrm{d})} ; h$ is the behav- ioral state during which firing rate values have been considered, MI is mutual information, and $\log$ is the logarithm in base 2 . Shuffled estimates were computed by randomly scrambling the order of firing rates for neuron $X$ and also scrambling their delayed version, $X^{(\mathrm{d})}$, in a different, random order. This does not affect marginal probability distributions $p(x \mid h)$ and $p\left(x^{(\mathrm{d})} \mid h\right)$, only the joint probability distribution $p\left(x, x^{(\mathrm{d})} \mid h\right)$. cDAMI was computed by varying time bin duration, number of amplitude bins, and delay $d$. cDAMI values for values of $d>0$ were normalized by the cDAMI value for $d=0$ to better compare cDAMI values across different neurons. Examples obtained for different time bin durations and delays can be seen in Figure 5, $A$ and $C$. cDAMI rapidly decreases to values close to 0 for values of $d>1$. We therefore only included cDAMI computed for $d=1$ in subsequent analyses. We found variations in cDAMI as a function of the behavioral state to depend neither on the duration of time bins nor on the number of amplitude bins. For consistency, we included in our subsequent analyses a similar range of time and amplitude bins as we used in the evaluation of cMI between areas, which we also based on mutual information. For each neuron, we computed the average cDAMI value for time bins ranging from 600 to $900 \mathrm{~ms}$ and a number of amplitude bins ranging from 10 to 20 . This range of values is outlined as a black rectangle in Figure 5, $A$ and $C$. Therefore, considering only a delay term $d=1$ corresponds to computing the average cDAMI for delays between 600 and $900 \mathrm{~ms}$.

Relationship between cDAMI and firing rates. To investigate whether cDAMI was monotonically related to the average firing rate of a neuron, we used a linear mixed-effects model to analyze the relationship between cDAMI values and average firing rates in the different behavioral states. We found the relationship to be non-significant ( $p=0.82$ ), so cDAMI is not related to the average spiking activity of a neuron.

\section{Statistical analysis}

Statistical analysis was performed via custom-made scripts in MATLAB (The MathWorks) and R (http://www.R-project.org). Because fewer neurons were recorded from V1M than from other areas, results obtained from V1 neurons were pooled together with those from S1BF neurons for statistical analyses related to Figures 4 and 5 (and referred to as neurons recorded in PRIM). Importantly, both cDAMI and cMI followed similar trends for neurons located in V1M and S1BF. For the analyses related to Figures 6, 7, and 8, we pooled together data from all areas and only subdivided them based on whether connections were within/between areas (see Figs. 6, 7) or between TM/non-TM neurons (see Fig. 8).

\section{Statistical analysis of information-theoretical measures}

Because all information-theoretical measures displayed highly skewed distributions, nonparametric tests were consistently used for comparing their values. The Friedman test with post hoc analysis was used to analyze whether cDAMI and cMI values varied as a function of behavioral state because each measure was evaluated separately for the same neuron or pair of neurons across AW, QW, and NREM. The Kruskal-Wallis test with post hoc analysis was used when an independent sample analysis had to be performed; for example, when comparing connections between different neuronal subpopulations.

\section{Statistical analysis of the proportion of significantly connected pairs of neurons}

A bootstrapping procedure was used to estimate confidence intervals ( $\alpha=0.05)$ for the proportion of cMI values that were significantly larger than 0 . For a given set of $n$ pairs of neurons, confidence intervals were obtained by sampling with replacement a new set of $n$ pairs of neurons and computing the proportion of significant $\mathrm{cMI}$ values for the resampled subset. The procedure was repeated 1000 times and the 2.fifth and 97.fifth percentile of the distribution of values were used as confidence intervals for the measured proportion. Proportions with nonoverlapping confidence intervals were considered to be significantly different.

\section{Results}

To investigate how neuron-level functional coupling is modulated across brain states, we used multi-area tetrode recordings 
A

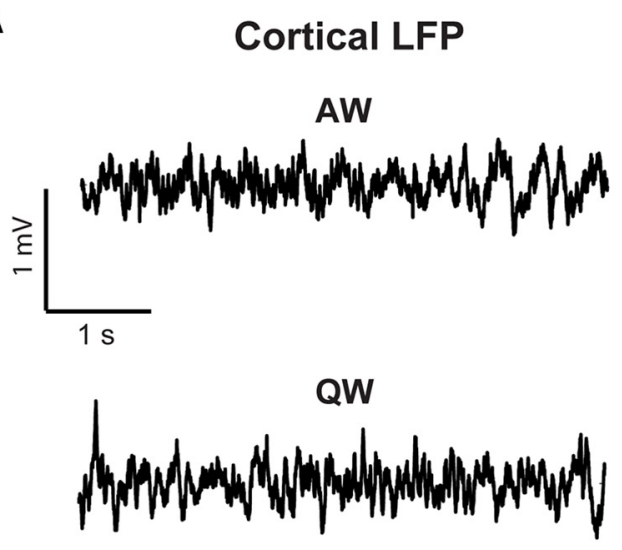

B

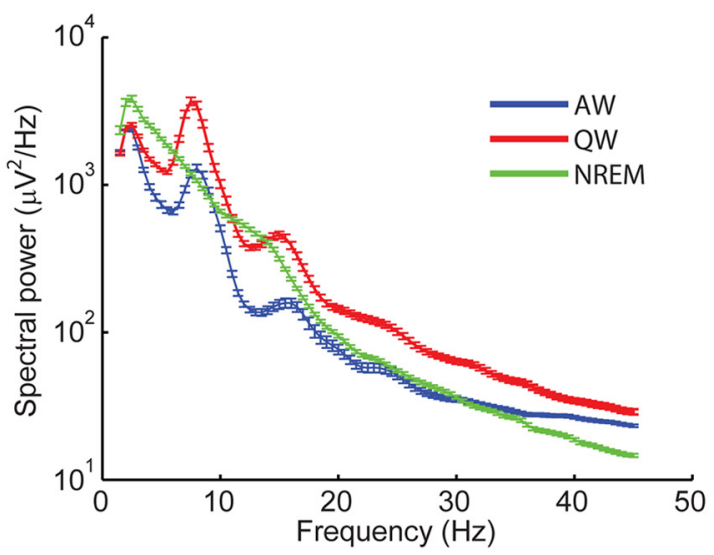

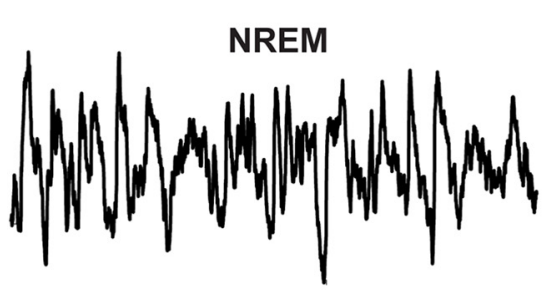

C
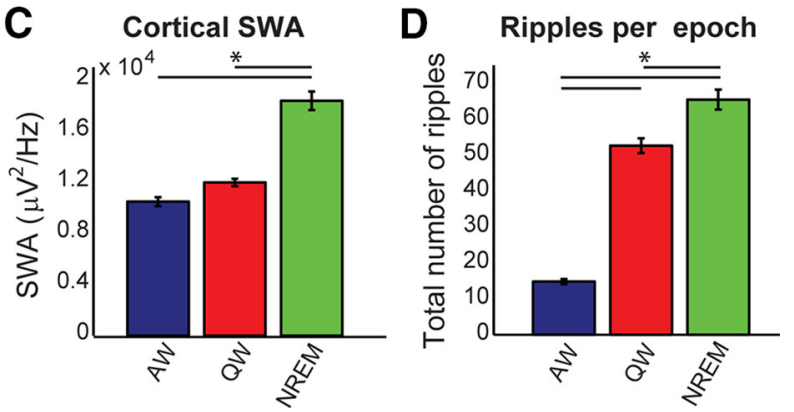

$\mathbf{E}$

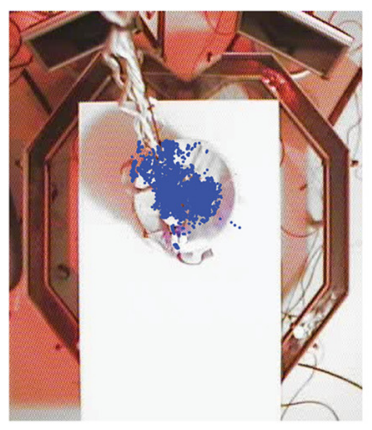

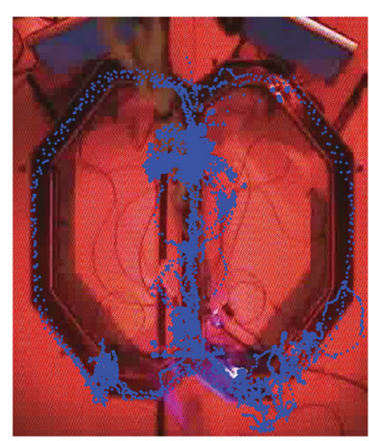

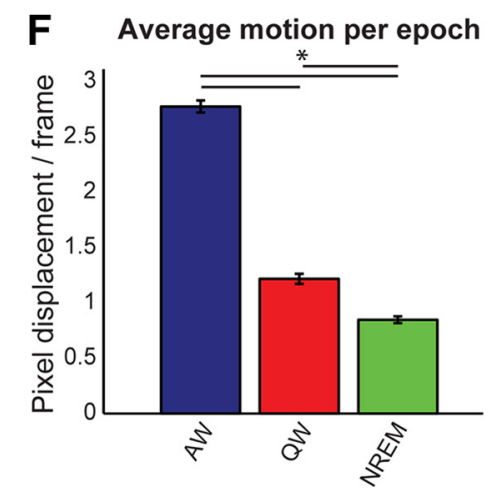

Figure 1. Scoring of behavioral states. $A$, Example LFP traces from one cortical channel (S1BF) recorded during (top to bottom) AW, QW, and NREM. Note the predominance of lower frequencies and higher amplitude during NREM. $\boldsymbol{B}$, Example single-session power spectra for the LFP channel shown in $\boldsymbol{A}$ during epochs characterized as AW (blue), QW (red), or NREM (green). In NREM, a theta peak is absent, whereas lower frequencies become more prominent. See the Materials and Methods for definitions of all criteria used to score behavioral states. $C$, Average SWA measured from epochs classified as AW, QW, or NREM, measured from the LFP channel shown in $\boldsymbol{A}$ and $\boldsymbol{B}$. Note the strong increase during NREM epochs. $\boldsymbol{D}$, Average total number of ripples reported from all hippocampal channels in each epoch (classified as AW, QW, or NREM). Ripples are mostly present in QW and NREM epochs. $E$, Example results from the motion tracking algorithm. The animal was either confined in a flower pot (left) or roaming in a figure-8 maze environment. Each blue dot represents the identified animal position in a video frame. $F$, Average body motion (measured as the average of pixel displacement for frames within single epochs) as a function of behavioral state (classified as AW, QW, or NREM). Motion is mostly confined to AW epochs, with remaining motion in $Q W$ and NREM being due to nonlocomotory activity (e.g., grooming) and noise level of the object tracking algorithm. Data are expressed as mean \pm SEM. Asterisks in $\boldsymbol{C}, \boldsymbol{D}$, and $\boldsymbol{F}$ indicate significant differences ( $p<0.05$, one-way ANOVA followed by post hoc Bonferroni correction).

(Lansink et al., 2007; Vinck et al., 2015b) to sample neuronal activity with single-cell resolution in primary sensory cortices (V1M and S1BF), PRH, and HPC simultaneously in freely moving rats. These areas are part of an anatomical and functional network for sensory processing, navigation, and episodic memory formation (Witter et al., 2000). Rats were trained to perform a sensory discrimination task in a figure- 8 maze (designed to modulate neuronal activity in all four recorded areas; see Materials and Methods). Recordings were conducted when animals were either performing the task or resting (see Materials and Methods; Fig. 1). Recording periods were scored into three behavioral states (AW, QW, and NREM sleep) based on electrophysiological and behavioral features (see Materials and
Methods; Fig. 1). Our ability to discriminate single-neuron activity allowed us to go beyond the currently available mesoscopic level of analysis and investigate connections between individual neurons in the same or different brain regions. Functional coupling was determined by using nonlinear measures based on information theory (MacKay, 2003) and specifically on mutual information (Steuer et al., 2002). In contrast to widely used linear methods such as correlation (Steuer et al., 2002; Pereda et al., 2005), mutual information and derived measures can quantify both linear and nonlinear coupling, the latter being a crucial feature of neuronal dynamics (Friston, 2000). Although most previous studies exploited information theory as a tool to assess stimulus-response relationships (Quian Quiroga and 
A

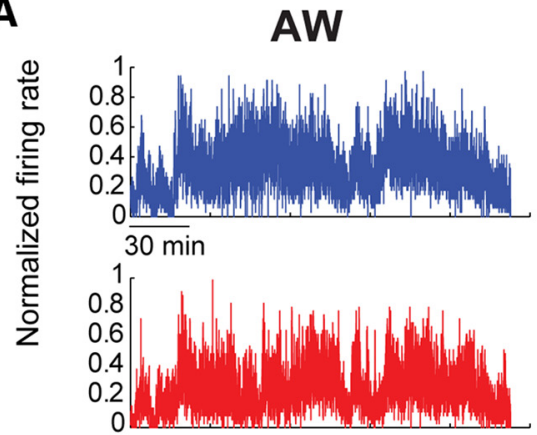

B

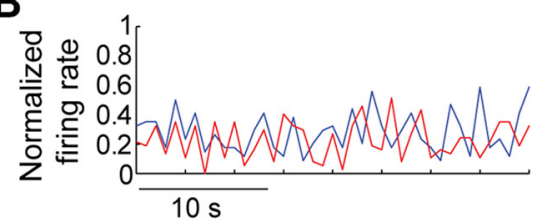

C

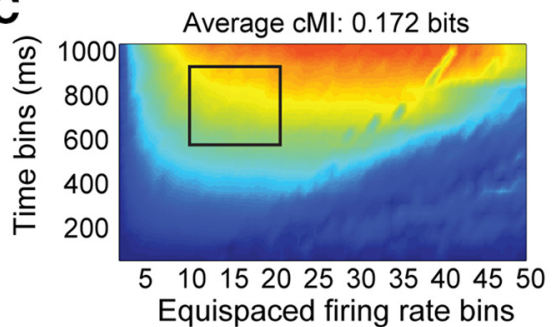

D

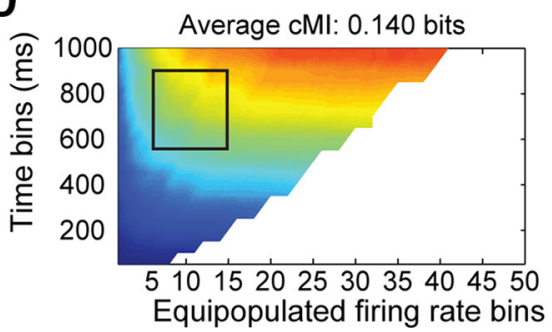

QW
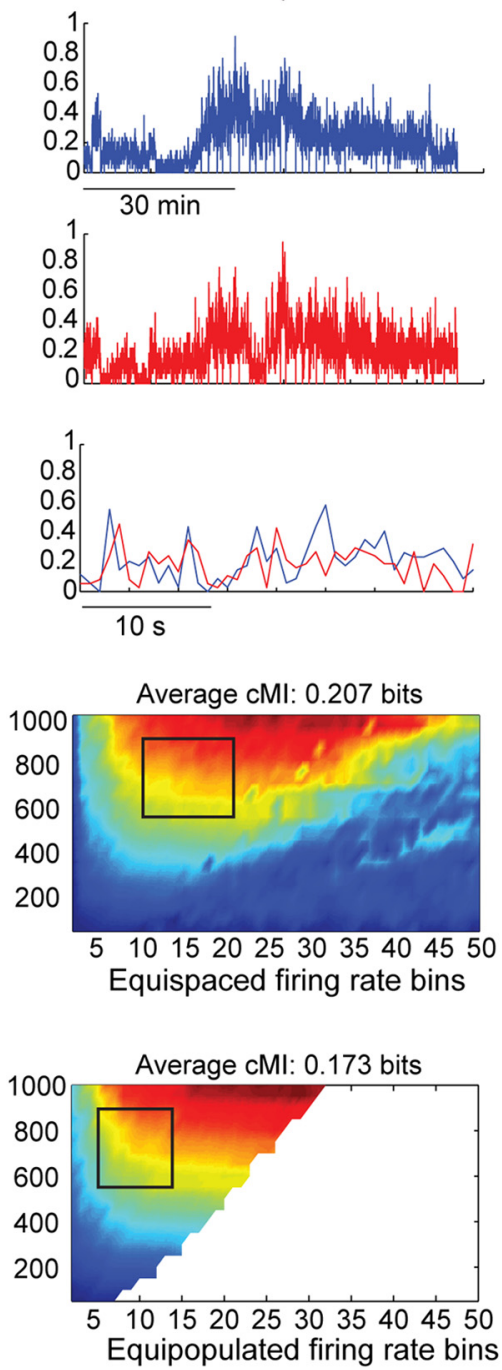

NREM
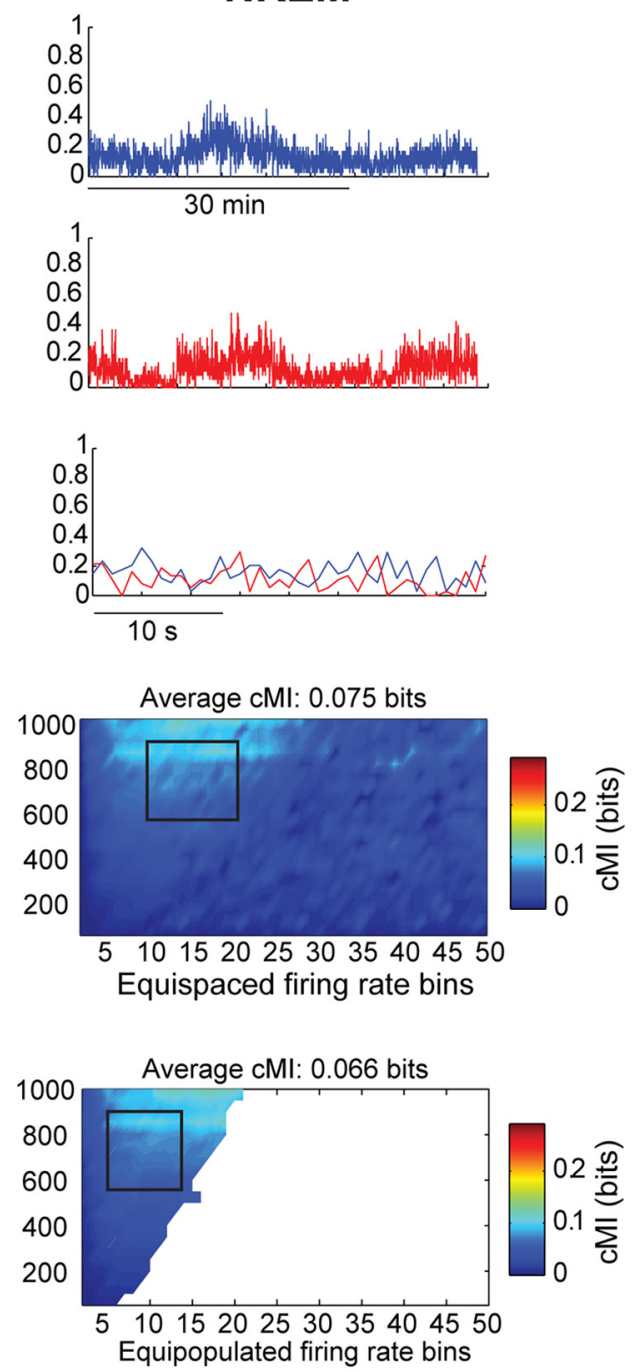

Figure 2. cMl values reflect coordinated firing rate fluctuations between neurons. $\boldsymbol{A}$, Example normalized firing rates ( $800 \mathrm{~ms}$ bin) for two example neurons (blue and red traces) across the three brain states (left: AW, middle: QW, right: NREM). All epochs classified as AW, QW, or NREM have been collapsed (i.e., drawn adjacent to each other). Firing rates for each neuron have been normalized to the highest firing rate recorded across all brain states. $\boldsymbol{B}$, Enlarged fragments of the firing rate traces shown in $\boldsymbol{A}$. $\boldsymbol{C}$, cMl values for the same example neuronal pair, computed using equispaced amplitude binning as a function of brain state, number of amplitude bins, and temporal bin duration. Each plot corresponds to one behavioral state (left: AW, middle: QW, right: NREM). cMI was computed for time bins of different durations ( $y$-axis, 50 to $1000 \mathrm{~ms}$ with a step size of $50 \mathrm{~ms}$ ) and for different amplitude bins ( $x$-axis, 2 to 50 bins). The black outline indicates the time bins and amplitude bins that were used in all further analyses (time bins: $600-900 \mathrm{~ms}$, amplitude bins: $10-20$ ). The average cMl value in the interval within the black outline is indicated in the header of each subplot. $\boldsymbol{D}$, Same as C but using equipopulated amplitude binning. White areas indicate ranges with fewer distinct firing rate values than number of bins for which no $\mathrm{cMl}$ value was estimated. The average $\mathrm{CMI}$ value in the interval within the black outline is indicated in the header of each subplot (time bins: $600-900$ ms, amplitude bins: 5-15). Note the similarity between the cMl values computed in $\boldsymbol{C}$ and $\boldsymbol{D}$ (see also Materials and Methods).

Panzeri, 2009), we used it here to determine coupling between individual neurons.

\section{Behavioral-state-dependent functional connectivity between} brain regions is regulated in a region-specific manner

Functional coupling between individual neurons was measured in terms of cMI (MacKay, 2003; see Materials and Methods). Specifically, we aimed to quantify how the coordination of firing rate fluctuations (Harris and Thiele, 2011) between individual neurons varies across brain states. Figure $2, A$ and $B$, shows two example neurons with firing rates that were co-modulated (i.e., they underwent similar variations over time). cMI was computed between pairs of neurons over a wide range of amplitude and temporal scales. Out of this extensive range, for all subsequent analyses, we selected a relevant narrower range in which we ob- served a significant state-dependent modulation (Figs. 2C, 4A; Materials and Methods). The selected range of time bins (600$900 \mathrm{~ms})$ and number of amplitude bins (10-20; see black outline in Figs. $2 C, 4 A$ ) enabled us to sample cMI values in an intermediate interval where we could avoid the loss of both sensitivity and generality (see Materials and Methods). Conversely, taking a range in the area where cMI values were close to zero vastly increased the uncertainty in our measures. We did not observe significant changes in the results of our analysis when the bin range was modified by \pm 2 time bins or \pm 5 amplitude bins. However, we cannot fully exclude that other combinations of time and amplitude bins might lead to different results. Importantly, results were fully compatible when we used either equispaced or equipopulated amplitude binning (Fig. 2C,D; Materials and Methods). 


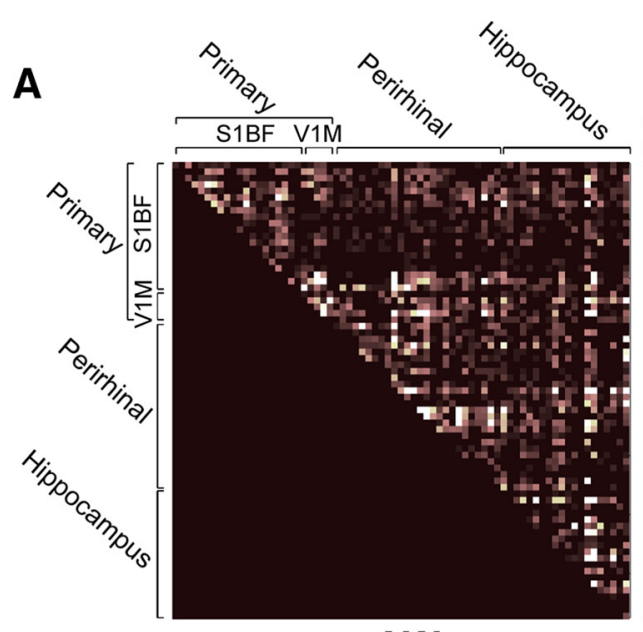

AW

\section{B}

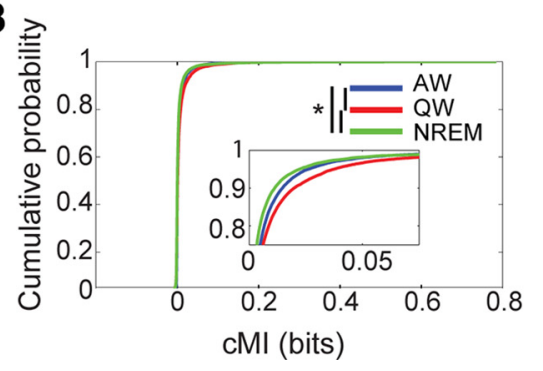

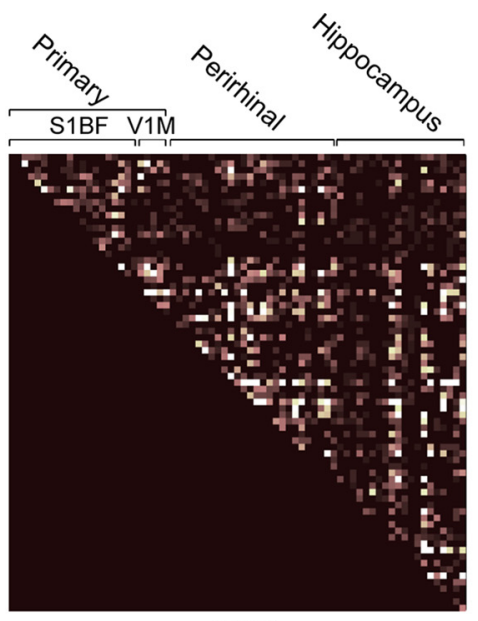

QW

C

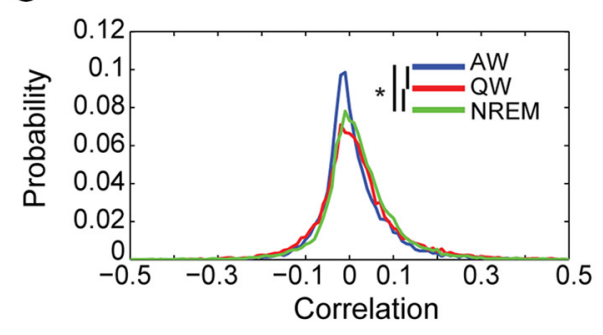

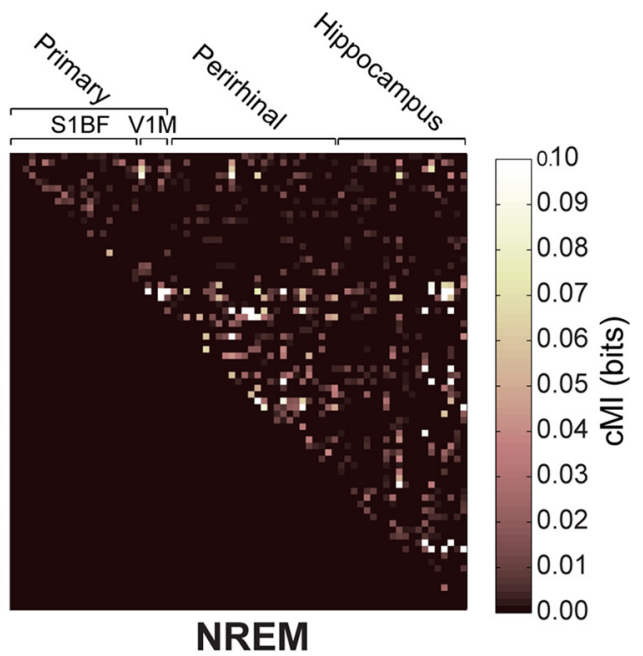

D

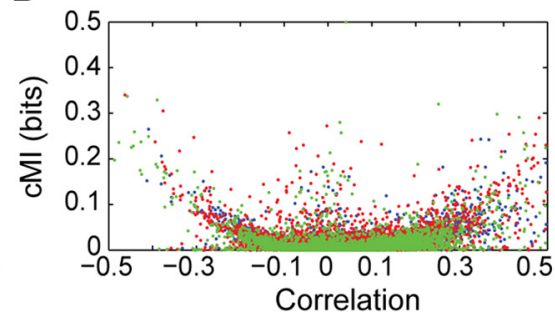

Figure 3. cMl values vary across behavioral states and are an extension of linear correlation values. $\boldsymbol{A}, \mathrm{cMl}$ values between individual neurons measured during a single recording session as a function of behavioral state. Brackets indicate in which brain areas neurons were located. Each row and column represent a single neuron. $\boldsymbol{B}$, Cumulative distribution plot for cMl values (all pairs of neurons in all brain regions and recording sessions) as a function of brain state (blue: AW, red: QW, green: NREM). Inset, Enlargement of the distributions around the 80th-100th percentiles. Nonparametric testing revealed significant differences between $\mathrm{CMI}$ values for all connections between the three behavioral states, with values being the largest for QW and the lowest for NREM $\left({ }^{*} p<0.05\right.$, Friedman test with post hoc analysis). C, Probability distribution of pairwise "linear" correlation values between trains of binned firing rates for all recorded neurons across brain states (blue: AW, red: QW, green: NREM). Firing rates were computed using an $800 \mathrm{~ms}$ time bin. Asterisks indicate significant differences between distributions ( $p<0.05$, Kolmogorov-Smirnov test with post hoc analysis). $\boldsymbol{D}$, Scatter plot showing the relationship between the pairwise correlation values of $\boldsymbol{B}$ and the cMl values of $\boldsymbol{C}$ as a function of brain state (blue: AW, red: QW, green: NREM).

Importantly, cMI is strongly related to the joint firing activity of pairs of neurons, an effect that cannot be explained from the individual firing rates of the neurons considered in the computation (see Materials and Methods). Furthermore, cMI during AW reflects interactions between neurons rather than spurious correlations arising from the modulation of firing rates after, for example, sensory stimuli or motor activity (see Materials and Methods). Although we found larger differences between NREM and wakefulness than within the two awake states, AW and QW (see also Figs. 5, 6, 7), these did show distinct traits and we therefore kept them separate in the following analyses.

Figure $3 A$ displays cMI between distinct pairs of neurons during a single recording session as a function of brain state. Note that $\operatorname{cMI}(X, Y)=c M I(Y, X)$, so the lower half of each matrix is left empty. During AW, most neuronal pairs showed non-zero cMI values; during QW, cMI values were generally higher, but more black spots started to appear, indicating a lower number of functionally interconnected neurons (i.e., of neurons with significantly correlated firing activities); during NREM, the proportion of black spots increased as a consequence of the decrease of significantly coupled neuronal pairs. This was also quantified over all recording sessions in Figure $3 B$. To better interpret the meaning of cMI values, we also measured the pairwise Pearson correlation coefficient between firing rates (Fig. 3C). We found that the correlation coefficients during NREM showed both stronger positive and negative correlations than during AW (and in par- ticular stronger positive correlations in the range between 0.1 and 0.2 ). This is consistent with the hypothesis that neurons can remain in a desynchronized state even when driven by a common oscillatory rhythm, as is the case for the neocortex in NREM (Harris and Thiele, 2011). This also indicates that the information conveyed by correlation coefficient and cMI values is not the same (as is evident by comparing the state-dependent changes in cMI and correlation values shown in Fig. $3 B, C$ ). We thus plotted, for each neuronal pair and each behavioral state, cMI values as a function of correlation values (Fig. 3D). This plot indicates a clear, nonlinear correspondence between cMI and correlation values, with a "U"-shaped distribution accompanied by points with significant $\mathrm{cMI}$ values, but correlations close to 0 . Compared with linear correlations, $\mathrm{cMI}$ is thus a more comprehensive, yet non-signed, measure of the strength of interdependencies between fluctuations of firing rate patterns.

To investigate whether the global brain-state dependency of cMI also showed local or interregional specificities, we pooled pairs of neurons based on the brain regions from which they were recorded. We then evaluated $\mathrm{cMI}$ values for each connection both within and between regions as a function of behavioral state (Fig. $4 B-D$ ). For these analyses, results for V1M were pooled together with those for S1BF (see Materials and Methods) and we refer to these neurons as being recorded in PRIM.

For intraneocortical connections, cMI peaked in QW and bottomed in NREM (Fig. $4 B, C$ ), thus confirming previous EEG results indicating a decrease in cortical integration during 

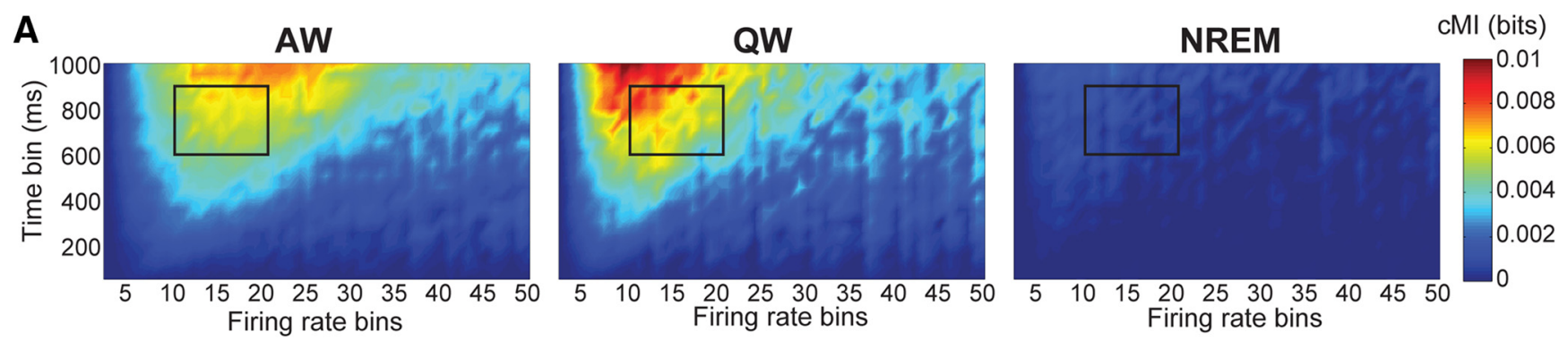

B

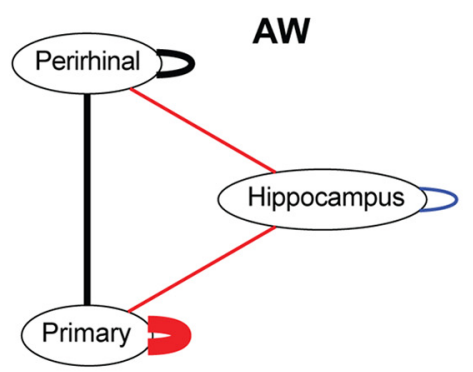

Median CMI

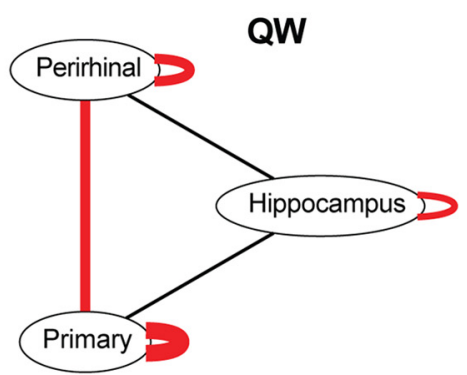

$1 \times 10^{-3}$ bits
$5 \times 10^{-3}$ bits

\section{C} $\times 10^{-3} \quad$ Median cMl between pairs of neurons

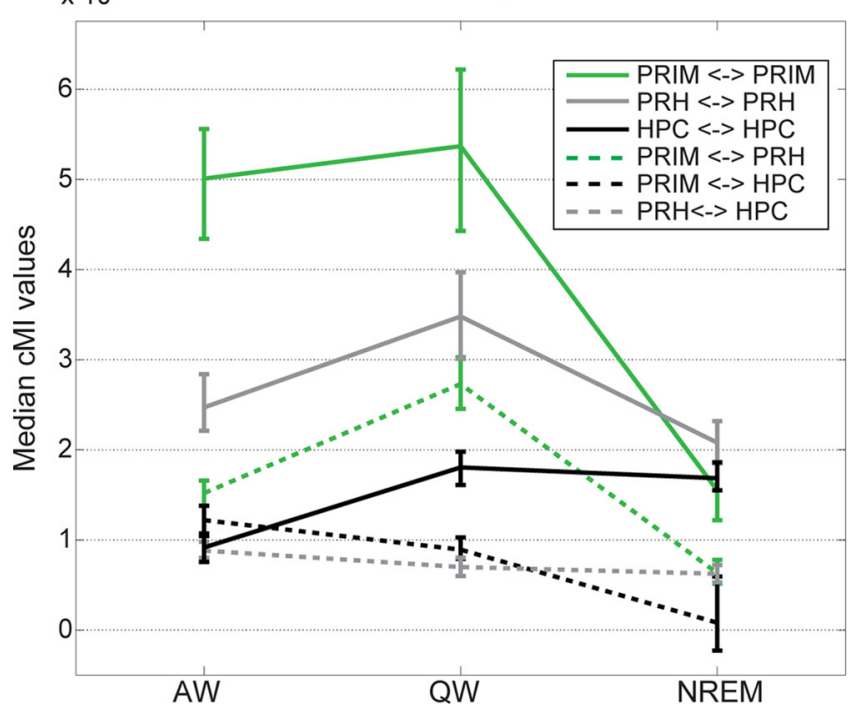

D

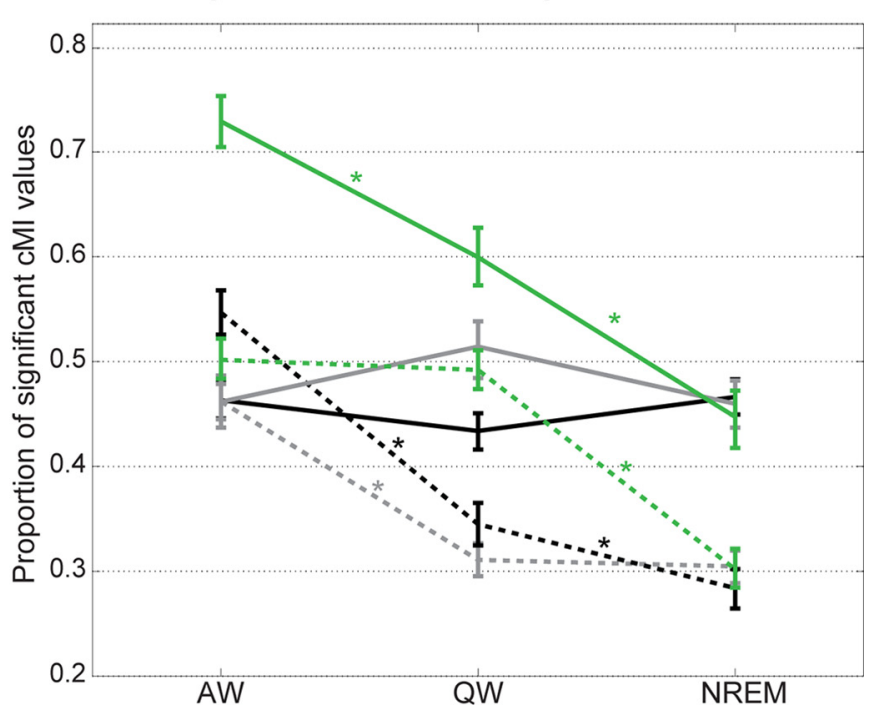

Figure 4. Functional connectivity between and within brain areas is modulated heterogeneously by behavioral state. $A$, Average values of $\mathrm{CMI}$ for pairs of neurons within $\mathrm{S} 1 \mathrm{BF}$ for a single recording session. Each plot corresponds to one behavioral state. cMI has been computed for time bins of different durations ( $y$-axis, 50 to $1000 \mathrm{~ms}$ with a step size of $50 \mathrm{~ms}$ ) and for different amplitude bins ( $x$-axis, 2 to 50 bins). The black outline indicates the time bins and amplitude bins that were used in all further analyses. $B$, Graphs of median cMl between and within regions as a function of behavioral state. The thickness of each connection is proportional to the CMI between or within a region (rounded to the closest multiple of $1 \times 10^{-3}$ bits; refer to $\mathrm{Cfor}$ non-approximated values). Absence of a connection indicates a median value not significantly larger than 0 (one-sided Wilcoxon test). Colors indicate the outcome of a Friedman test with post hoc analysis performed separately for each set of connections across behavioral states. Red indicates that a connection was significantly stronger in one or more specific behavioral states than in all others. Blue indicates that a connection was significantly weaker in one or more specific behavioral states than in all others. Black indicates an intermediate value. $\boldsymbol{C}$, Same as in $\boldsymbol{B}$, indicating the non-approximated values of median cMl for each connection as a function of the behavioral state. Error bars indicate confidence intervals for the medians. For significant differences across behavioral states, see $\boldsymbol{B}$. $\boldsymbol{D}$, Plot indicating the proportion of neuronal pairs for which cMl values are significantly larger than 0 ( $p<0.05$, based on the distribution of shuffled estimates, see Materials and Methods). Each line corresponds to a specific connection between or within areas. Error bars are bootstrap-estimated confidence intervals (see Materials and Methods). Asterisks indicate significant differences across behavioral states for each type of connection ( $p<0.05$, bootstrap-estimated).

NREM (Massimini et al., 2005). In addition, cMI for corticohippocampal connections peaked during AW, whereas cMI for intrahippocampal connections bottomed in AW. This indicates that behavioral states modulate functional connectivity between brain regions in a region-specific manner. Although, on average, cMI is higher in wakefulness than in NREM, differences exist between cortical areas and hippocampus (Fig. 4B). Specifically, cMI within the neocortex peaked in QW and bottomed in NREM; cMI within the hippocampus also peaked in QW, but remained relatively strong in NREM and bottomed in AW; finally, cMI between the neocortex and hippocampus peaked in AW and bottomed in NREM. 
Behavioral state modulated not only cMI values but also the proportion of pairs of neurons displaying cMI values that were significantly larger than 0 (Fig. 4D; Materials and Methods). Although median cMI values generally peaked during QW (with the exception of cortico-hippocampal connections; Fig. 4B,C), we found that the proportion of significant cMI values was on average larger in AW and progressively lower in QW and NREM (Fig. 4D). Only for connections within the perirhinal cortex and within the hippocampus was no significant change across behavioral states observed.

Both cMI values and the proportion of significant values during NREM did not markedly change when excluding time bins containing ripples from our analysis (see Materials and Methods). Although ripples have been implicated in information transfer between cortex and hippocampus (Pennartz et al., 2009; Pezzulo et al., 2014; Buzsáki, 2015), cMI only quantifies coordinated, simultaneous fluctuations in firing rates and thus cannot capture such directional, short-term influences.

Overall, these results indicate a state-dependent modulation of functional connectivity between neurons. On average, connections between and within brain areas were found to be stronger and denser in wakefulness than in NREM. Specific differences were found between AW and QW and for intrahippocampal and cortico-hippocampal connections. Therefore, functional connectivity between neurons varies in a regionally specific and statedependent manner both in terms of connection strength and proportion of actively coupled neurons.

\section{Recurrent connectivity in cortico-hippocampal circuits is weaker in NREM sleep than in wakefulness}

We next investigated whether the same behavioral-state dependency and regional heterogeneity that we found for functional (undirected) connectivity between pairs of neurons was also present for reentrant (or self-) connections at the level of single neurons (Edelman and Gally, 2013). Recurrent connectivity is a key factor in the generation of a balanced and functional cortical activity (Douglas et al., 1995; Haider et al., 2006) and has been proposed to be a key component of conscious processing (Balduzzi and Tononi, 2008; Pennartz, 2009; Edelman and Gally, 2013). Reentrant connectivity was measured by cDAMI (Pompe et al., 1998; MacKay, 2003; Materials and Methods; Fig. 5A,C), a nonlinear measure that quantifies how strongly the current activity of a neuron influences its future activity. Specifically, cDAMI measures recurrent functional connectivity as the mutual information between the activity of a neuron at time $t$ and the activity of the same neuron at time $t+1$ as a function of behavioral state. cDAMI does not discriminate between autaptic (monosynaptic self-feedback) and polysynaptic feedback onto a single neuron, and between these and intrinsic firing properties (e.g., bursting). An example cDAMI plot for a single neuron is shown in Figure $5 A$ and its nonlinear relationship with the neuron's activity can be seen by comparing cDAMI plots with the auto-correlogram (Barthó et al., 2004) shown in Figure 5B. The autocorrelogram for the displayed example neuron shows higher values during AW than both QW and NREM (with slightly higher values for QW compared with NREM) for time scales longer than $50 \mathrm{~ms}$, and this rank order is nonlinearly mirrored by the cDAMI values computed using the corresponding temporal bins (cf. Fig. $5 A, B)$. Both the autocorrelograms and cDAMI show a prominent drop for time scales shorter than $50 \mathrm{~ms}$. Here, as was the case for cMI, we focused on time bins in the order of hundreds of milliseconds. cDAMI is therefore a measure of how firing rate fluctuations over time scales in the order of hundreds of millisec- onds persist at the level of single neurons. cDAMI was averaged over the same range of time bins and number of amplitude bins that we used for cMI (see Materials and Methods).

We hypothesized that recurrent functional connectivity would be stronger during conscious states (Shu et al., 2003) (AW and QW) compared with unconscious ones (NREM). We found that, in all recorded regions, cDAMI values were lower in NREM than in both AW and QW (Fig. 5D, $p<0.05$, Friedman test with post hoc analysis). In the hippocampus, cDAMI was also significantly lower in QW than in AW. Overall, these results indicate that, at the level of single neurons, a given pattern of neural activity more quickly dissipates during NREM than during wakefulness, a further sign of the decay in integration during NREM.

\section{Decrease in inter-areal coupling between excitatory but not inhibitory neurons, and preservation of intra-area connectivity during NREM sleep}

Next, we investigated the cell-level mechanisms responsible for the global drop of neuronal coupling that we observed during NREM sleep. We discriminated putative excitatory neurons from putative interneurons based on the shape of action potential waveforms (Barthó et al., 2004; Iurilli et al., 2013; Fig. 6A; Materials and Methods). Specifically, putative inhibitory neurons display a narrower action potential waveform than putative excitatory neurons. We confirmed previous reports that average firing rates are lower during NREM than during awake states. In particular, we found firing rates to be significantly higher for excitatory neurons in AW, and progressively lower in QW and NREM (Vyazovskiy et al., 2009; Fig. 6 B, C, left). We also evaluated how the distribution of firing rate values shifted within each neuronal subtype as a function of brain state. To this aim, we normalized, separately for each neuron and brain state, firing rates to the highest recorded value (Fig. $6 B, C$, right). As expected from the literature (Steriade et al., 2001), NREM sleep is characterized by an increase in low firing rates at the expense of higher ones for both excitatory and inhibitory neurons. A bimodal distribution, representing an alternation between up and down states, was not observed here, probably for two reasons: (1) we binned spike trains without discriminating between active and silent periods, so bins, which were several hundreds of milliseconds long, could include both; and (2) most neurons we recorded displayed low firing rates (Fig. $6 B, C$, left), thus increasing the chance of observing silent periods also during AW and QW. QW showed distinct patterns of normalized firing rate distributions for excitatory and inhibitory neurons (Fig. $6 B, C$, right). For the former, firing rates were shifted to values higher than those observed in AW; for inhibitory neurons, the firing rate distribution in QW was similar to that present in NREM.

Reentrant connections (cDAMI) were weaker in NREM than in wakefulness for both excitatory and inhibitory neurons (Fig. $6 D$ ), indicating a generalized drop of recurrent functional connectivity in NREM.

We next shifted our focus to investigating coupling between pairs of excitatory or inhibitory neurons. First, we evaluated how correlations between pairs of excitatory or inhibitory neurons varied as a function of brain state (Fig. 6E). Pairs of excitatory neurons showed the highest proportion of both positive and negative correlation values in QW, especially higher positive ones, followed by NREM and AW (Fig. 6E, left). For pairs of inhibitory neurons, the only significant difference was between AW and NREM (Fig. 6E, right), the latter being associated with a wider distribution of correlation values. 
A

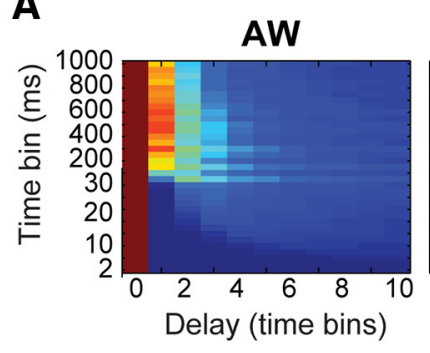

C

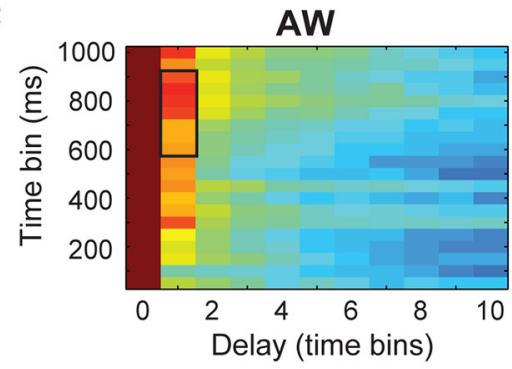

D

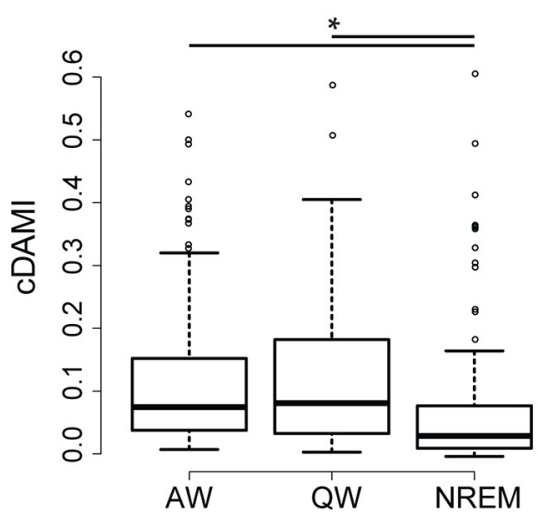

QW

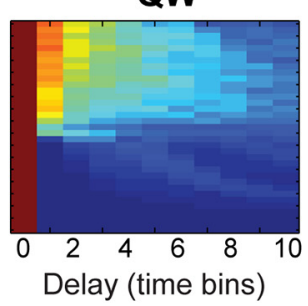

NREM

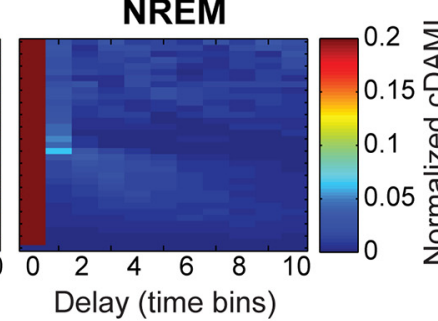

B

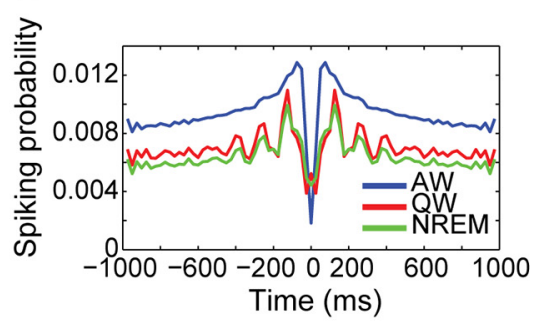

QW

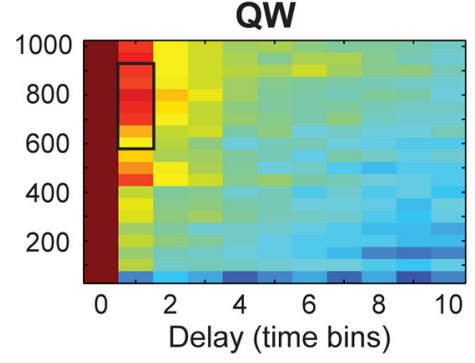

Perirhinal cortex

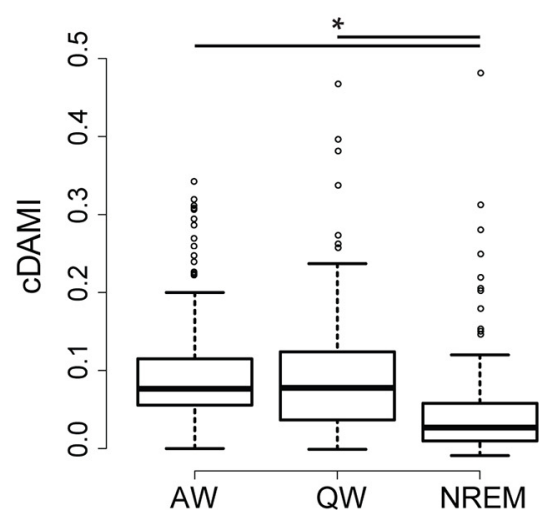

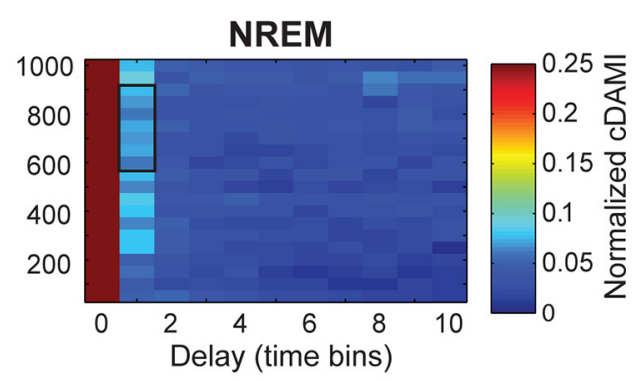

Hippocampus

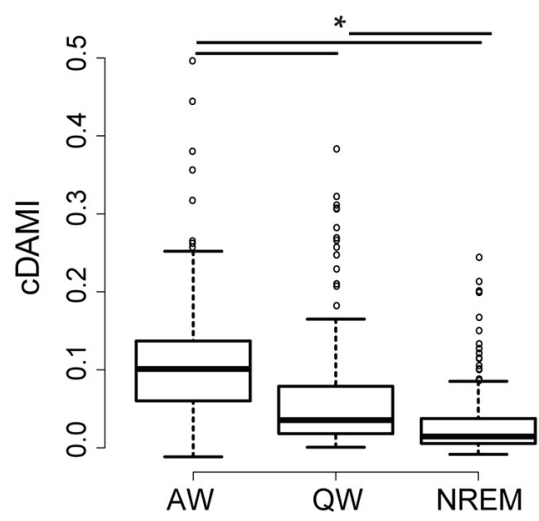

Figure 5. Recurrent connectivity is heterogeneously modulated by behavioral state. A, CDAMI plot for a single example neocortical neuron. Each plot corresponds to one behavioral state. CDAMI has been computed for time bins of different durations ( $y$-axis, 2 to $1000 \mathrm{~ms}$ ), for different delay bins ( $x$-axis), and for different time bin widths (the duration of one delay bin is equal to the corresponding time bin width given on the $y$-axis; e.g., a delay of 1 time bin for a time bin width of $600 \mathrm{~ms}$ is exactly $600 \mathrm{~ms}$ ). For each time bin width, cDAMI for delay bins $>0$ is normalized to the corresponding value with no delay (see Materials and Methods). $\boldsymbol{B}$, Auto-correlogram (probability of one spike by a neuron being followed or preceded by a spike by the same neuron) for the neuron shown in $A$ computed using a $25 \mathrm{~ms}$ bin in the range -1 to $1 \mathrm{~s}$ for each behavioral state (blue: AW, red: QW, green: NREM). $C$, Average values of CDAMI across neurons in the barrel cortex for a single recording session. Each plot corresponds to one behavioral state. CDAMI has been computed as described for $\boldsymbol{A}$ (for time bins ranging between 50 and $1000 \mathrm{~ms}$ ). The black outline indicates the values of time bins and delays that were used in all further analyses. $D$, Boxplots indicating the average values of $C D A M I$ for all recorded neurons (see Materials and Methods) in the three different brain regions that we considered. Asterisks indicate significant differences $(p<0.05$, Friedman test with post hoc analysis).

Nonlinear coupling (measured in terms of cMI) between pairs of excitatory neurons or pairs of inhibitory neurons was, like correlations, differently modulated by behavioral state (Fig. $6 F, G$ ). Although cMI between pairs of excitatory neurons decreased going from AW to QW and to NREM (both in terms of values and proportion of connected couples), cMI between interneurons did not vary and was stronger than that observed between excitatory neurons (Fig. 6G). These results indicate that, overall, the brain becomes more disconnected in NREM than during wakefulness, but this is primarily due to a decrease in the coupling between pyramidal neurons, not of interneurons.

We then investigated the spatial nature of the decrease in network connectivity during NREM sleep by analyzing pairs of neurons separately within and between distinct brain areas. We found that functional connectivity between excitatory neurons located in different areas decreased going from wakefulness (AW and QW) to NREM, both in terms of cMI values and proportion of connected couples (Fig. 7A, B, left). Conversely, only minor changes were observed between excitatory neurons located in the same area and between interneurons (Fig. $7 A, B$ ); specifically, no consistent difference was found for $\mathrm{cMI}$ values and proportion of significantly connected couples between NREM and both AW and QW (see the legends of Fig. 7A,B for details). Therefore, whereas, during NREM, connectivity at the local level (i.e., single-area) and between interneurons is preserved, communication between excitatory neurons located in distinct brain areas diminishes.

The pattern observed for cMI closely resembled the differences between distributions of pairwise correlations for excitatory and inhibitory neurons located in the same or in different brain areas (Fig. $7 C$ ), with weaker inter-area than intra-area correlations during QW and NREM for pairs of excitatory neurons, but no difference for pairs of inhibitory neurons. 

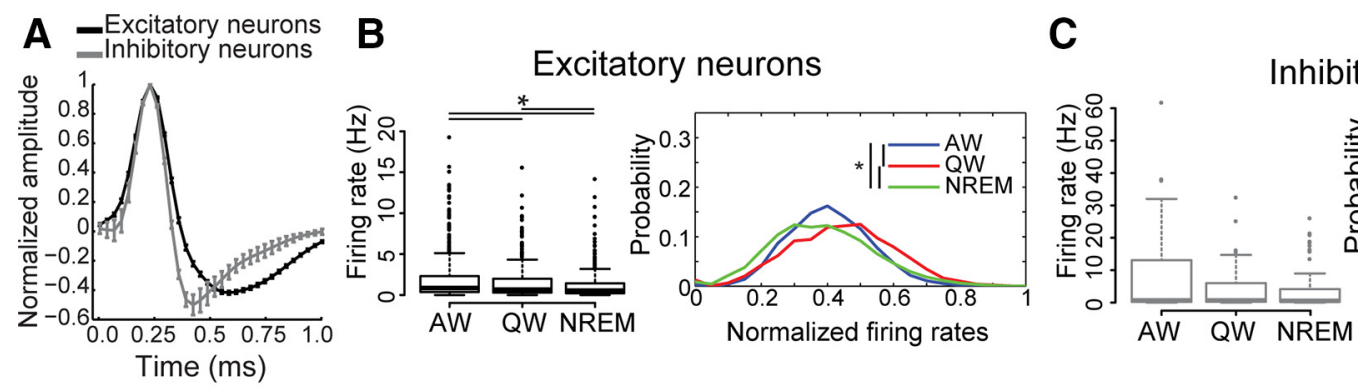

Inhibitory neurons
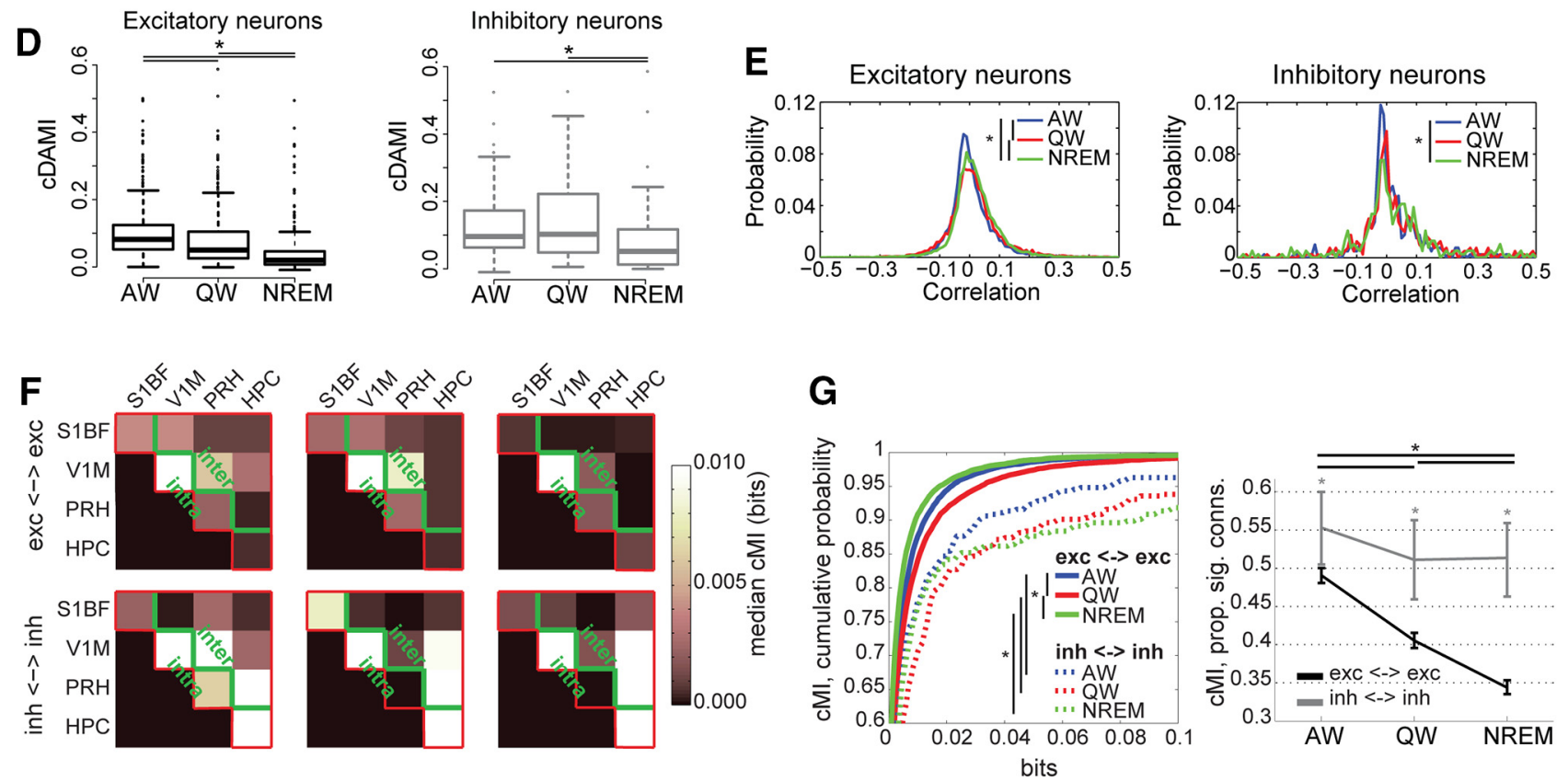

Figure 6. Differential modulation of functional connectivity between excitatory and inhibitory neurons as a function of behavioral state. $A$, Average action potential waveforms (mean \pm SEM) for neurons classified as either broad-spiking (putative excitatory neurons, black) or fast-spiking (putative inhibitory neurons, gray). $\boldsymbol{B}$, Left, Firing rates for putative excitatory neurons across brain states. Asterisks indicate significant differences ( $p<0.05$, Friedman test with post hoc analysis). Right, Distribution of normalized firing rates for excitatory neurons only as a function of brain state (blue: AW, red: QW, green: NREM) computed using an $800 \mathrm{~ms}$ time bin. Firing rates within each brain state have been normalized (independently for each neuron) to the highest value recorded across all three brain states. Asterisks indicate significant differences between distributions across behavioral states ( $p<0.05$, Wilcoxon rank-sum test with post hoc analysis). $\boldsymbol{C}$, Same as $\boldsymbol{B}$ but for inhibitory neurons. D, Boxplots of CDAMI for putative excitatory neurons (left, black) and putative inhibitory neurons (right, gray) as a function of behavioral state. For both excitatory and inhibitory neurons, CDAMI is weaker in NREM than in AW and QW, with CDAMI being larger in AW than QW for excitatory neurons (asterisks indicate $p<0.05$, Friedman test with post hoc analysis). For all behavioral states, CDAMI was larger for inhibitory than for excitatory neurons $(p<0.05$, Mann-Whitney $U$ test). $E$, Probability distribution of pairwise linear correlation values between firing rate trains separately for all recorded excitatory (left) or inhibitory (right) neurons, across brain states (blue: AW, red: QW, green: NREM). Firing rates were computed using an $800 \mathrm{~ms}$ time bin. Asterisks indicate significant differences between distributions across behavioral states ( $p<0.05$, Kolmogorov-Smirnov test with post hoc analysis). $\boldsymbol{F}$, Median cMl values within and between brain areas measured during a single recording session as a function of behavioral state. Red lines indicate the border between computed cMl values and empty portion of the matrix (see also Figure $3 A$ ). Top, Median cMl values between pairs of excitatory neurons. Bottom, Median CMI values between pairs of inhibitory neurons. Green lines indicate the boundary between intra-area and inter-area connections (below and above the green line, respectively). G, Left, Cumulative probability distribution curves of CMI values as a function of behavioral state for pairs of putative excitatory neurons (solid lines) and pairs of putative inhibitory neurons (dashed lines) pooled across all areas for different behavioral states (blue: AW, red: QW, green: NREM). Asterisks indicate significant differences ( $p<0.05$, Friedman test with post hoc analysis). Right, Proportion of connections significantly larger than $0(p<0.05)$ for the different types of neuronal pairs (black: pairs of excitatory neurons; gray: pairs of inhibitory neurons) as a function of behavioral state. Error bars indicate bootstrap-estimated confidence intervals (see Materials and Methods). Asterisks and lines at the top of each panel indicate significant differences across behavioral states ( $p<0.05$, bootstrap-estimated, only one asterisk for all lines); line colors and styles correspond to the connection to which they refer, as indicated in the legend; asterisks on top of error bars indicate significant differences between types of connections within each behavioral state.

Task-related functional networks remain segregated during quiet wakefulness and NREM sleep

All analyses presented so far are independent from the previous history of neuronal activity. Although it is widely accepted that neuronal activity occurring during wakefulness strongly influences firing patterns during quiet wakefulness and sleep (reactivation of stored memory traces; Foster and Wilson, 2006; Ji and Wilson, 2007; Pezzulo et al., 2014), only a few studies have investigated whether functional multi-area networks, as defined by involvement (or non-involvement) in a behavioral task, display consistent patterns of functional connectivity across different brain states (Hoffman and McNaughton, 2002; Ji and Wilson, 2007; Lansink et al., 2009). We first investigated differences in network dynamics based on whether neuronal firing rates were significantly modulated by the task (Fig. 8A,B; Materials and Methods). We found that cDAMI was significantly larger for non-TM than for TM neurons during QW and NREM, but not AW (Fig. 8C).

In contrast, functional connectivity (cMI) showed features different from those of cDAMI. During AW, cMI between TM neurons was found to be larger than cMI between non-TM neurons and between one TM and one non-TM neuron, in terms of 
A

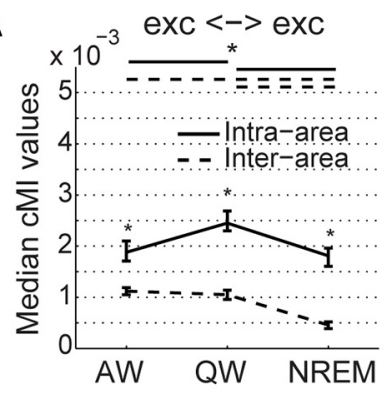

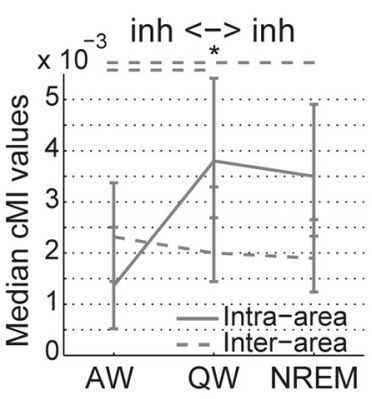
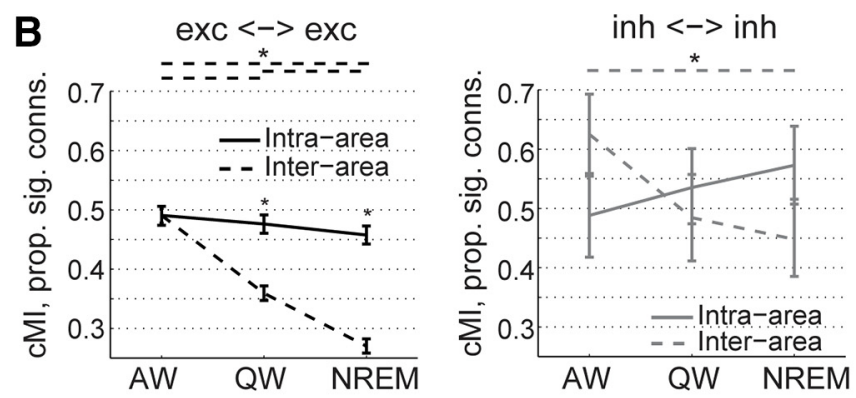

C
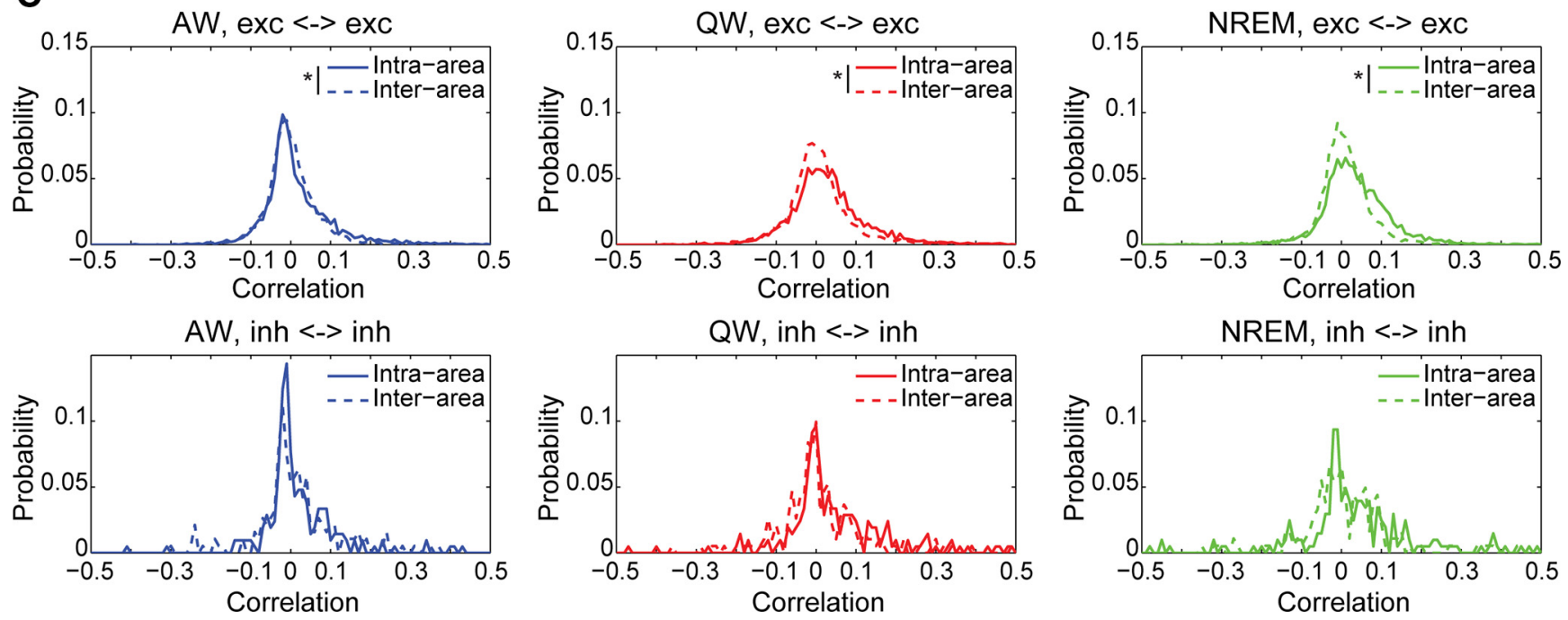

Figure 7. Differential state-dependent modulation of functional connectivity between excitatory and inhibitory neurons for intra-area versus inter-area connections. $A$, Median cMl values as a function of behavioral state between different types of neuronal pairs based on whether neurons were located in the same brain area or in different areas (solid lines: intra-area connections; dashed lines: inter-area connections). Error bars indicate confidence intervals for the medians. Left, black, cMl between pairs of excitatory neurons; for inter-area connections, cMI values during NREM were significantly lower than during both AW and QW; for intra-area connections, CMI values during NREM were lower than during QW but not AW. Right, gray, cMI between pairs of inhibitory neurons; the only significant difference was observed for inter-areal connections, for which cMI values during AW were lower than during both QW and NREM. Asterisks and lines at the top of each panel indicate significant differences across behavioral states ( $p<0.05$, Kruskal-Wallis test with post hoc analysis); line colors and styles correspond to the connection to which they refer, as indicated in the legend; asterisks on top of error bars indicate significant differences between types of connections within each behavioral state ( $p<0.05$, Wilcoxon rank-sum test). $\boldsymbol{B}$, Proportions of significant $(p<0.05) \mathrm{cMl}$ values for different types of connection as a function of behavioral state and based on whether neurons were located in the same or different areas (solid line: intra-area connections; dashed line: inter-area connections). Left, black, Pairs of excitatory neurons; no significant change was observed across behavioral states for intra-area connections, whereas a significant progressive decrease change was found for inter-area connections going from AW to QW and NREM. Right, gray, Pairs of inhibitory neurons; the only significant difference was present for inter-areas connections between AW and NREM (but not between QW and NREM). Error bars are bootstrap-estimated confidence intervals (see Materials and Methods). Asterisks and lines at the top of each panel indicate significant differences across behavioral states $(p<0.05$, bootstrap-estimated, only one asterisk for all lines); line colors and styles correspond to the connection to which they refer, as indicated in the legend; asterisks on top of error bars indicate significant differences between types of connections, within each behavioral state. C, Probability distribution of pairwise correlation values between firing rate trains as a function of brain state (blue, left: AW; red, middle: QW; green, right: NREM), neuronal subtype (top: excitatory neuron; bottom: inhibitory neurons), and type of connection (solid lines: intra-area; dashed lines: inter-area). Asterisks indicate significant differences between types of connection within brain state and neuronal subtype ( $p<0.05$, Kolmogorov-Smirnov test).

both values and proportion of significant values (Fig. 8D,E). During QW and NREM, instead, we found that cMI (again, for both value and proportion of significant connections) was lower for connections between a TM and a non-TM neuron than for connections between pairs of TM neurons or pairs of non-TM neurons, with no difference between the latter two types of connection (Fig. $8 D, E$ ). This indicates that, during QW and NREM (i.e., in brain states having no direct bearing on the task), TM and non-TM neurons continue to behave as distinct functional networks that interact weakly with each other.

\section{Discussion}

Although behavioral states have long been considered global phenomena involving the whole brain (Pace-Schott and Hobson, 2002), several recent experiments indicate that neural activity patterns during different behavioral states have a highly heterogeneous nature (Hirase et al., 2001b; Pennartz et al., 2002; Klaus- berger et al., 2003; Greenberg et al., 2008; Vyazovskiy et al., 2009, 2011; Gentet et al., 2010; Sachidhanandam et al., 2013; Goltstein et al., 2015). Surprisingly, investigation of state-dependent modulation of brain connectivity has been mostly limited to the macroscopic (Massimini et al., 2005; Ferrarelli et al., 2010; Park and Friston, 2013) and mesoscopic (Lewis et al., 2012; Lu et al., 2012; Bettinardi et al., 2015; Pigorini et al., 2015) scales. Here, we provide an account of how brain state modulates functional connectivity between individual neurons in the rat neocortex and hippocampus. Specifically, we quantified the coordination between firing rate fluctuations over hundreds of milliseconds (Harris and Thiele, 2011). Compared with faster neuronal processes, usually referred to as assembly or packet activity and occurring over periods of 30-200 ms (Miller et al., 2014; Luczak et al., 2015; Montijn et al., 2015), such slower fluctuations are thought to modulate (or gate) how effectively infor- 
A
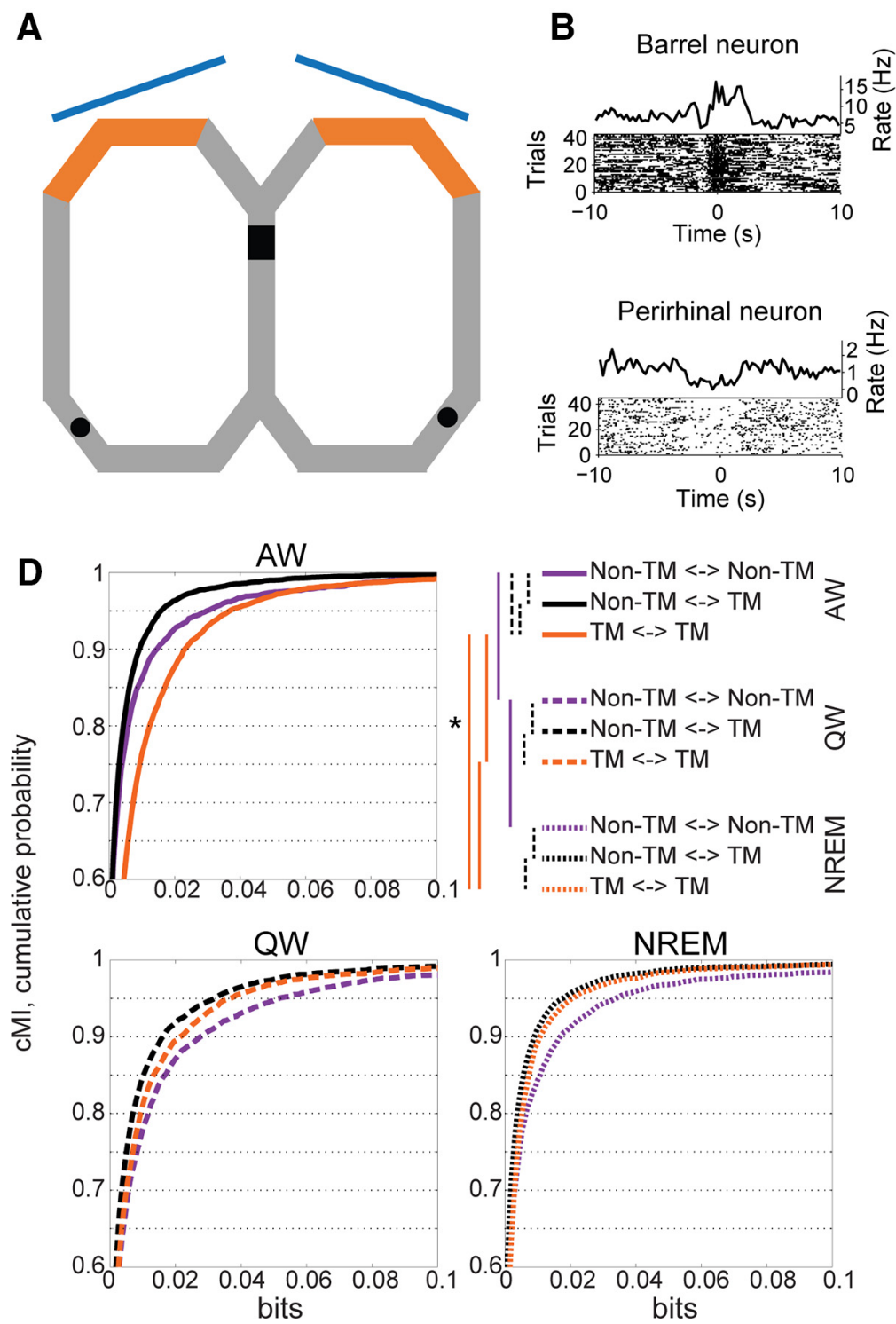

C TM neurons

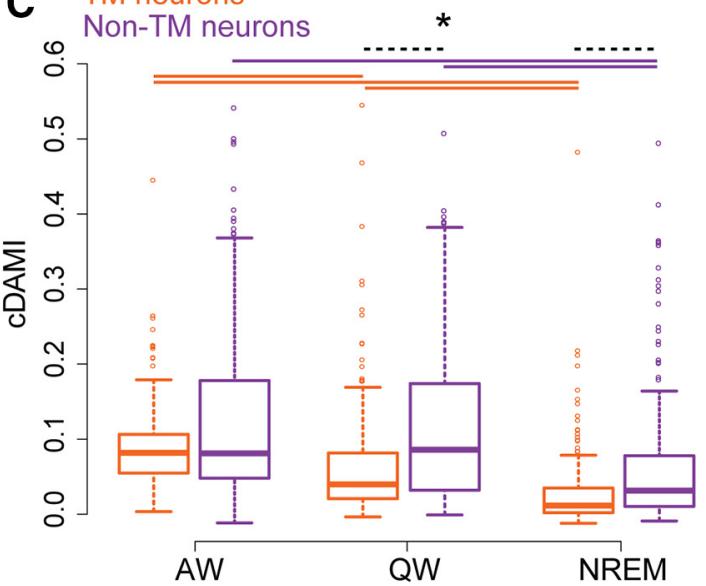

$\mathbf{E}$

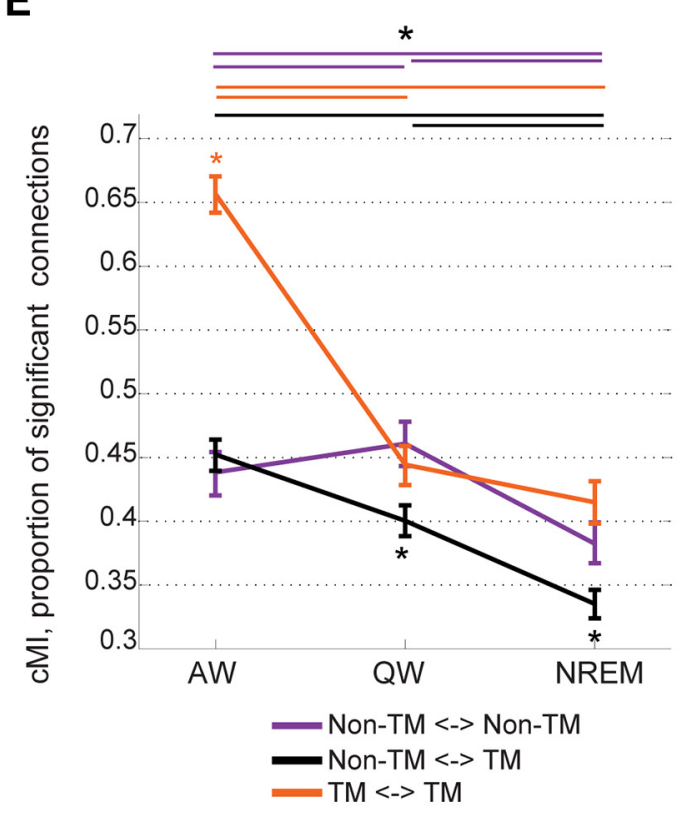

Figure 8. Task-related networks remain segregated in quiet wakefulness and NREM sleep. $A$, Outline of maze (gray track) and behavioral task. Black rectangle indicates the starting point of each trial, where visual stimuli (blue: screens) were observed. Orange areas are the maze portion with sandpaper on side walls. Black areas are the reward locations. $\boldsymbol{B}$, PETHs and raster plots for two example TM neurons. Top, Neuron in S1BF (time 0: entrance in the sandpaper zone). Bottom, Neuron in PRH (time 0: reward delivery). C, cDAMI for neurons of which the firing rate was either modulated (TM, orange traces) or not (non-TM neurons, purple traces) during task performance. Horizontal lines (with one asterisk for all lines): significant differences across behavioral states (solid lines: orange for TM neurons, purple for non-TM neurons; Friedman test with post hoc analysis) and between TM and non-TM neurons within each behavioral state (dashed black lines: differences between TM and non-TM within a single state, $p<0.05$, Wilcoxon rank-sum test). $\boldsymbol{D}$, Cumulative probability distribution of cMl values during the different behavioral states as a function of the type of neuronal pair: pairs of non-TM neurons (purple curves), pairs of one non-TM and one TM neuron (black curves), and pairs of TM neurons (orange curves). Top left, AW (solid lines). Bottom left, QW (dashed lines). Bottom right, NREM (dotted lines). Top right, Dashed lines: significant differences within each behavioral state ( $p<0.05$, Kruskal-Wallis test with post hoc analysis). Solid lines indicate significant differences across behavioral states ( $p<0.05$, Friedman test with post hoc analysis) for each type of neuronal pair (indicated by the corresponding color). $\boldsymbol{E}$, Proportion of $c \mathrm{MI}$ values significantly larger than 0 for the different types of pairs of neurons (orange: pairs of TM neurons; purple: pairs of non-TM neurons; black: one non-TM and one TM neuron) as a function of behavioral state. Error bars are bootstrap-estimated confidence intervals. Colored asterisk above (or below) all lines at single brain states indicate the connection with the significantly $(p<0.05$ ) highest (lowest) proportion of positive cMI values. Horizontal lines (with one asterisk for all lines) indicate significant differences across behavioral states for each type of neuronal pair (colors correspond to neuronal pair types, $p<0.05$, bootstrap-estimated).

mation can be transferred between neurons, rather than the "message" to be processed (Luczak et al., 2013). We found that, on average, NREM is associated with a lower level of network integration compared with wakefulness (both AW and QW), as indicated by cMI and cDAMI (Figs. 2, 3, 4, and 5). However, we also found that: (1) functional connectivity within and between brain areas is modulated across behavioral states in a region-specific manner, with differences between neocortex and hippocampus (Figs. 2, 3, 4, 5); (2) functional coupling between interneurons and connectivity within single areas are not significantly modulated by behavioral states, but inter-areal coupling between excitatory neurons is reduced in NREM (Figs. 6F, G, 7A,B); and (3) functional networks formed by TM and non-TM neurons remain segregated during QW and NREM (Fig. 8D,E). Our general conclusion is that the different stages of wakefulness and sleep are global behavioral phenomena with specific local neural correlates. This had so far been observed only at the level of single-cell spiking activity (Gentet et al., 2010; Vyazovskiy et al., 2011). For example, local clusters of cortical neurons can enter sleep- 
like states independently from the rest of the cortex (Vyazovskiy et al., 2011) and individual neuronal subtypes were shown to have specific brain-state dependent patterns of activity (Gentet et al., 2010; Vinck et al., 2015b). Our findings indicate that, similar to firing activity, neuronal coupling is also tightly regulated at the single-neuron level.

During NREM, inter-area functional connectivity between excitatory neurons was diminished, whereas coupling was preserved at the level of individual areas and between interneurons (Figs. 6, 7). This indicates a potential mechanism underlying the breakdown of neural integration, which has been described during unconscious states (anesthesia and NREM) at the macroscale (Massimini et al., 2005; Ferrarelli et al., 2010), and fragmentation of activity shown to occur at the mesoscale (Lewis et al., 2012; Pigorini et al., 2015). Therefore, whereas global brain integration is lost during NREM, it is preserved at the local level and individual brain areas might, at least during depolarized states, preserve a minimal level of activity to allow fast reengagement in conscious processing (Destexhe et al., 2007).

Our results reveal potential functional differences between excitatory and inhibitory neurons across the sleep-wake cycle. Preserved coupling between interneurons and distancedependent decline between excitatory neurons during NREM was described previously at the single-area level in humans (Peyrache et al., 2012). Here, we found that functional connectivity between interneurons is also preserved between different brain areas during NREM (Fig. $7 A, B$ ). This was confirmed by our analysis of pairwise correlations between neurons located in same or different areas (Fig. 7C). Compared with linear methods (such as pairwise correlations), however, cMI captures a broader range of interrelationships between firing rate fluctuations (e.g., nonmonotonic dependencies). Therefore, although the synchronous occurrence of up and down states during NREM likely leads to higher pairwise linear correlations (Figs. 3C, 6E), this is accompanied by a stronger drop in nonlinear coupling (Figs. 3B, 4C).

Overall, our results indicate that, during NREM, interneurons help to maintain an adequate level of coupling between brain areas, for example, by synchronizing rhythmic activity within and between areas (Caputi et al., 2013). Conversely, excitatory neurons are primarily involved in communication, defined as information transfer between areas (Olcese et al., 2013), and functional connectivity between them declines when less information is transferred, possibly due to a decline in functional connectivity between excitatory neurons, as observed in NREM. Our results thus support the view that interneurons might be involved in modulating oscillatory activity during NREM to foster coordination (i.e., coupling without information transfer) between brain areas (Puig et al., 2008; Kilduff et al., 2011). What could be the mechanisms underlying preserved coupling between interneurons during NREM? Although interneurons with longrange connections have been described (Melzer et al., 2012) and could play a role, common subcortical inputs may also constitute a major driving force for interneuron synchronization: specifically, direct neocortical projections from both the thalamus (Contreras et al., 1997) and the basal forebrain (Jones, 2004; Henny and Jones, 2008). Measures of functional coupling such as cMI cannot discriminate between these two possible mechanisms: future studies using methods to quantify effective (directional) connectivity such as transfer entropy (Schreiber, 2000) and optogenetics will be necessary to address this point.

Brain state also modulated functional connectivity depending on the involved brain regions, with a major difference between neocortex and hippocampus. Within the neocortex, both cMI and CDAMI peaked in wakefulness and bottomed in NREM, mirroring the key role of the neocortex in conscious processing. Intrahippocampal coupling followed instead a different trend: whereas cDAMI peaked in AW and was progressively lower in QW and NREM, cMI peaked in QW and was lower in AW than NREM (Figs. 4C, 5D). Therefore, during AW, hippocampal neurons show high level of self- but not mutual connectivity and the opposite occurs in NREM. Future experiments and analyses will be required to determine whether this is conducive to establishing the role of the hippocampus in episodic memory formation and sleep-dependent consolidation (McNaughton et al., 1983; Lee and Wilson, 2002; Pennartz et al., 2002; Girardeau and Zugaro, 2011).

Finally, we found that TM neurons and non-TM neurons form distinct functional networks not only, as expected, during AW (Tauste Campo et al., 2015), but also during QW and NREM. We observed that CMI between pairs of TM-neurons or between pairs of non-TM neurons was stronger than between one TM and one non-TM neuron during QW and NREM (Fig. 8D,E). This functional segregation between TM and non-TM neurons may be due to within-network (i.e., within TM or non-TM neurons) correlated firing activity and consequential Hebbian learning, which would selectively strengthen connections between TM (or non-TM) neurons, but not between different functional networks (Olcese et al., 2010). Importantly, this learning process may have occurred over the course of the whole training period (i.e., several weeks) and this may explain why we observed functional segregation during QW and NREM periods, which occurred both before and after AW. Segregation between networks with different functional profiles might be one of the mechanisms underlying the coordinated and selective reactivation of patterns of neuronal firing across brain areas in NREM sleep (Hoffman and McNaughton, 2002; Ji and Wilson, 2007; Lansink et al., 2009; Olcese et al., 2010), which must avoid the involvement of neurons unrelated to the replayed experience. We also observed that cDAMI for TM neurons was lower than cDAMI for non-TM neurons in QW and NREM, but not AW (Fig. 8C). Low cDAMI can indicate both weaker recurrent connectivity and faster dynamics because it quantifies how strongly patterns of activity at the level of single neurons dissipate over time (Fig. 5A,C). Reactivation of patterns of spiking activity during QW and NREM occurs is time compressed compared with task performance (e.g., navigation) in AW (Hoffman and McNaughton, 2002; Lee and Wilson, 2002; Foster and Wilson, 2006; Euston et al., 2007; Ji and Wilson, 2007; Lansink et al., 2009; Pezzulo et al., 2014). This is consistent with lower cDAMI values for TM-neurons during $\mathrm{QW}$ and NREM because faster, yet highly correlated, dynamics would result in a decrease in cDAMI at the temporal scales that we measured. However, it remains to be determined whether timecompressed sleep replay contributes to the lower cDAMI of TM neurons.

In conclusion, we provide for the first time an account of how inter-area and intra-area coupling vary as a function of behavioral state at a cellular level. Our analysis demonstrates a highly local and interregional nature for brain-state-dependent modulation of neural coupling. Importantly, whereas inter-areal coupling between excitatory neurons is reduced in NREM compared with wakefulness, functional connectivity within single areas and between interneurons is preserved. Future studies will be needed to generalize our findings to other brain regions, locations within single areas (e.g., cortical layers), temporal scales, and identified 
neuronal phenotypes and to understand the functional roles of this modulation.

\section{Notes}

Supplemental material for this article is available at http://hdl.handle. net/11245/2.174271 (online-only material, including nine additional figures). This material has not been peer reviewed.

\section{References}

Arabzadeh E, Panzeri S, Diamond ME (2004) Whisker vibration information carried by rat barrel cortex neurons. J Neurosci 24:6011-6020. CrossRef Medline

Balduzzi D, Tononi G (2008) Integrated information in discrete dynamical systems: motivation and theoretical framework. PLoS Comput Biol 4:e1000091. CrossRef Medline

Barthó P, Hirase H, Monconduit L, Zugaro M, Harris KD, Buzsáki G (2004) Characterization of neocortical principal cells and interneurons by network interactions and extracellular features. J Neurophysiol 92:600-608. CrossRef Medline

Bastianini S, Berteotti C, Gabrielli A, Lo Martire V, Silvani A, Zoccoli G (2015) Recent developments in automatic scoring of rodent sleep. Arch Ital Biol 153:58-66. Medline

Bettinardi RG, Tort-Colet N, Ruiz-Mejias M, Sanchez-Vives MV, Deco G (2015) Gradual emergence of spontaneous correlated brain activity during fading of general anesthesia in rats: Evidences from fMRI and local field potentials. Neuroimage 114:185-198. CrossRef Medline

Buzsáki G (2015) Hippocampal sharp wave-ripple: A cognitive biomarker for episodic memory and planning. Hippocampus 25:1073-1188. CrossRef Medline

Caputi A, Melzer S, Michael M, Monyer H (2013) The long and short of GABAergic neurons. Curr Opin Neurobiol 23:179-186. CrossRef Medline

Contreras D, Destexhe A, Steriade M (1997) Intracellular and computational characterization of the intracortical inhibitory control of synchronized thalamic inputs in vivo. J Neurophysiol 78:335-350. Medline

Destexhe A, Hughes SW, Rudolph M, Crunelli V (2007) Are corticothalamic "up" states fragments of wakefulness? Trends Neurosci 30:334-342. CrossRef Medline

Douglas RJ, Koch C, Mahowald M, Martin KA, Suarez HH (1995) Recurrent excitation in neocortical circuits. Science 269:981-985. CrossRef Medline

Edelman GM, Gally JA (2013) Reentry: a key mechanism for integration of brain function. Front Integr Neurosci 7:63. Medline

Euston DR, Tatsuno M, McNaughton BL (2007) Fast-forward playback of recent memory sequences in prefrontal cortex during sleep. Science 318: 1147-1150. CrossRef Medline

Ferrarelli F, Massimini M, Sarasso S, Casali A, Riedner BA, Angelini G, Tononi G, Pearce RA (2010) Breakdown in cortical effective connectivity during midazolam-induced loss of consciousness. Proc Natl Acad Sci U S A 107:2681-2686. CrossRef Medline

Foster DJ, Wilson MA (2006) Reverse replay of behavioural sequences in hippocampal place cells during the awake state. Nature 440:680-683. CrossRef Medline

Franken P, Dijk DJ, Tobler I, Borbély AA (1991) Sleep deprivation in rats: effects on EEG power spectra, vigilance states, and cortical temperature. Am J Physiol 261:R198-R208. Medline

Franken P, Chollet D, Tafti M (2001) The homeostatic regulation of sleep need is under genetic control. J Neurosci 21:2610-2621. Medline

Friston KJ (2000) The labile brain. I. Neuronal transients and nonlinear coupling. Philos Trans R Soc Lond B Biol Sci 355:215-236. CrossRef Medline

Fuhrmann F, Justus D, Sosulina L, Kaneko H, Beutel T, Friedrichs D, Schoch S, Schwarz MK, Fuhrmann M, Remy S (2015) Locomotion, Theta Oscillations, and the Speed-Correlated Firing of Hippocampal Neurons Are Controlled by a Medial Septal Glutamatergic Circuit. Neuron 86:12531264. CrossRef Medline

Gentet LJ, Avermann M, Matyas F, Staiger JF, Petersen CCH (2010) Membrane potential dynamics of GABAergic neurons in the barrel cortex of behaving mice. Neuron 65:422-435. CrossRef Medline

Girardeau G, Zugaro M (2011) Hippocampal ripples and memory consolidation. Curr Opin Neurobiol 21:452-459. CrossRef Medline

Goltstein PM, Montijn JS, Pennartz CM (2015) Effects of isoflurane anes- thesia on ensemble patterns of Ca2 + activity in mouse v1: reduced direction selectivity independent of increased correlations in cellular activity. PloS One 10:e0118277. CrossRef Medline

Gourévitch B, Eggermont JJ (2007) Evaluating information transfer between auditory cortical neurons. J Neurophysiol 97:2533-2543. CrossRef Medline

Greenberg DS, Houweling AR, Kerr JND (2008) Population imaging of ongoing neuronal activity in the visual cortex of awake rats. Nat Neurosci 11:749-751. CrossRef Medline

Haider B, Duque A, Hasenstaub AR, McCormick DA (2006) Neocortical network activity in vivo is generated through a dynamic balance of excitation and inhibition. J Neurosci 26:4535-4545. CrossRef Medline

Harris KD, Shepherd GMG (2015) The neocortical circuit: themes and variations. Nat Neurosci 18:170-181. CrossRef Medline

Harris KD, Thiele A (2011) Cortical state and attention. Nat Rev Neurosci 12:509-523. CrossRef Medline

Hatsopoulos NG, Ojakangas CL, Paninski L, Donoghue JP (1998) Information about movement direction obtained from synchronous activity of motor cortical neurons. Proc Natl Acad Sci U S A 95:15706-15711. CrossRef Medline

Henny P, Jones BE (2008) Projections from basal forebrain to prefrontal cortex comprise cholinergic, GABAergic and glutamatergic inputs to pyramidal cells or interneurons. Eur J Neurosci 27:654-670. CrossRef Medline

Hirase H, Leinekugel X, Csicsvari J, Czurkó A, Buzsáki G (2001a) Behaviordependent states of the hippocampal network affect functional clustering of neurons. J Neurosci 21:RC145. Medline

Hirase H, Leinekugel X, Czurkó A, Csicsvari J, Buzsáki G (2001b) Firing rates of hippocampal neurons are preserved during subsequent sleep episodes and modified by novel awake experience. Proc Natl Acad Sci U S A 98:9386-9390. CrossRef Medline

Hoffman KL, McNaughton BL (2002) Coordinated reactivation of distributed memory traces in primate neocortex. Science 297:2070-2073. CrossRef Medline

Iurilli G, Olcese U, Medini P (2013) Preserved excitatory-inhibitory balance of cortical synaptic inputs following deprived eye stimulation after a saturating period of monocular deprivation in rats. PLoS One 8:e82044. CrossRef Medline

Ji D, Wilson MA (2007) Coordinated memory replay in the visual cortex and hippocampus during sleep. Nat Neurosci 10:100-107. CrossRef Medline

Jones BE (2004) Activity, modulation and role of basal forebrain cholinergic neurons innervating the cerebral cortex. Prog Brain Res 145:157-169. CrossRef Medline

Kilduff TS, Cauli B, Gerashchenko D (2011) Activation of cortical interneurons during sleep: an anatomical link to homeostatic sleep regulation? Trends Neurosci 34:10-19. CrossRef Medline

Klausberger T, Magill PJ, Márton LF, Roberts JD, Cobden PM, Buzsáki G, Somogyi P (2003) Brain-state- and cell-type-specific firing of hippocampal interneurons in vivo. Nature 421:844-848. CrossRef Medline

Kudrimoti HS, Barnes CA, McNaughton BL (1999) Reactivation of hippocampal cell assemblies: effects of behavioral state, experience, and EEG dynamics. J Neurosci 19:4090-4101. Medline

Lansink CS, Bakker M, Buster W, Lankelma J, van der Blom R, Westdorp R, Joosten RN, McNaughton BL, Pennartz CM (2007) A split microdrive for simultaneous multi-electrode recordings from two brain areas in awake small animals. J Neurosci Methods 162:129-138. CrossRef Medline

Lansink CS, Goltstein PM, Lankelma JV, McNaughton BL, Pennartz CM (2009) Hippocampus leads ventral striatum in replay of place-reward information. PLoS Biol 7:e1000173. CrossRef Medline

Lansink CS, Goltstein PM, Lankelma JV, Pennartz CM (2010) Fast-spiking interneurons of the rat ventral striatum: temporal coordination of activity with principal cells and responsiveness to reward. Eur J Neurosci 32: 494-508. CrossRef Medline

Lee AK, Wilson MA (2002) Memory of sequential experience in the hippocampus during slow wave sleep. Neuron 36:1183-1194. CrossRef Medline

Lewis LD, Weiner VS, Mukamel EA, Donoghue JA, Eskandar EN, Madsen JR, Anderson WS, Hochberg LR, Cash SS, Brown EN, Purdon PL (2012) Rapid fragmentation of neuronal networks at the onset of propofolinduced unconsciousness. Proc Natl Acad Sci U S A 109:E3377-E3386. CrossRef Medline 
Lu H, Zou Q, Gu H, Raichle ME, Stein EA, Yang Y (2012) Rat brains also have a default mode network. Proc Natl Acad Sci U S A 109:3979-3984. CrossRef Medline

Luczak A, Bartho P, Harris KD (2013) Gating of sensory input by spontaneous cortical activity. J Neurosci 33:1684-1695. CrossRef Medline

Luczak A, McNaughton BL, Harris KD (2015) Packet-based communication in the cortex. Nat Rev Neurosci 16:745-755. CrossRef Medline

MacKay DJC (2003) Information Theory, Inference and Learning Algorithms. Cambridge, UK; New York: Cambridge UP.

Magri C, Whittingstall K, Singh V, Logothetis NK, Panzeri S (2009) A toolbox for the fast information analysis of multiple-site LFP, EEG and spike train recordings. BMC Neurosci 10:81. CrossRef Medline

Makino H, Komiyama T (2015) Learning enhances the relative impact of top-down processing in the visual cortex. Nat Neurosci 18:1116-1122. CrossRef Medline

Massimini M, Ferrarelli F, Huber R, Esser SK, Singh H, Tononi G (2005) Breakdown of cortical effective connectivity during sleep. Science 309: 2228-2232. CrossRef Medline

McGinley MJ, David SV, McCormick DA (2015a) Cortical membrane potential signature of optimal states for sensory signal detection. Neuron 87:179-192. CrossRef Medline

McGinley MJ, Vinck M, Reimer J, Batista-Brito R, Zagha E, Cadwell CR, Tolias AS, Cardin JA, McCormick DA (2015b) Waking state: rapid variations modulate neural and behavioral responses. Neuron 87:1143-1161. CrossRef Medline

McNaughton BL, Barnes CA, O'Keefe J (1983) The contributions of position, direction, and velocity to single unit activity in the hippocampus of freely-moving rats. Exp Brain Res 52:41-49. Medline

Melzer S, Michael M, Caputi A, Eliava M, Fuchs EC, Whittington MA, Monyer H (2012) Long-range-projecting GABAergic neurons modulate inhibition in hippocampus and entorhinal cortex. Science 335: 1506-1510. CrossRef Medline

Miller JE, Ayzenshtat I, Carrillo-Reid L, Yuste R (2014) Visual stimuli recruit intrinsically generated cortical ensembles. Proc Natl Acad Sci U S A 111:E4053-E4061. CrossRef Medline

Montijn JS, Goltstein PM, Pennartz CM (2015) Mouse V1 population correlates of visual detection rely on heterogeneity within neuronal response patterns. eLife 4:e10163. CrossRef Medline

Olcese U, Esser SK, Tononi G (2010) Sleep and synaptic renormalization: a computational study. J Neurophysiol 104:3476-3493. CrossRef Medline

Olcese U, Iurilli G, Medini P (2013) Cellular and synaptic architecture of multisensory integration in the mouse neocortex. Neuron 79:579-593. CrossRef Medline

Pace-Schott EF, Hobson JA (2002) The neurobiology of sleep: genetics, cellular physiology and subcortical networks. Nat Rev Neurosci 3:591-605. CrossRef Medline

Panzeri S, Senatore R, Montemurro MA, Petersen RS (2007) Correcting for the sampling bias problem in spike train information measures. J Neurophysiol 98:1064-1072. CrossRef Medline

Park HJ, Friston K (2013) Structural and functional brain networks: from connections to cognition. Science 342:1238411. CrossRef Medline

Pennartz CM (2009) Identification and integration of sensory modalities: neural basis and relation to consciousness. Conscious Cogn 18:718-739. CrossRef Medline

Pennartz CM, Uylings HB, Barnes CA, McNaughton BL (2002) Memory reactivation and consolidation during sleep: from cellular mechanisms to human performance. Prog Brain Res 138:143-166. CrossRef Medline

Pennartz CM, Lee E, Verheul J, Lipa P, Barnes CA, McNaughton BL (2004) The ventral striatum in off-line processing: ensemble reactivation during sleep and modulation by hippocampal ripples. J Neurosci 24:6446-6456. CrossRef Medline

Pennartz CM, Berke JD, Graybiel AM, Ito R, Lansink CS, van der Meer M, Redish AD, Smith KS, Voorn P (2009) Corticostriatal Interactions during Learning, Memory Processing, and Decision Making. J Neurosci 29: 12831-12838. CrossRef Medline

Pereda E, Quiroga RQ, Bhattacharya J (2005) Nonlinear multivariate analysis of neurophysiological signals. Prog Neurobiol 77:1-37. CrossRef Medline

Peyrache A, Dehghani N, Eskandar EN, Madsen JR, Anderson WS, Donoghue JA, Hochberg LR, Halgren E, Cash SS, Destexhe A (2012) Spatiotemporal dynamics of neocortical excitation and inhibition during human sleep. Proc Natl Acad Sci U S A 109:1731-1736. CrossRef Medline
Pezzulo G, van der Meer MA, Lansink CS, Pennartz CM (2014) Internally generated sequences in learning and executing goal-directed behavior. Trends Cogn Sci 18:647-657. CrossRef Medline

Pigorini A, Sarasso S, Proserpio P, Szymanski C, Arnulfo G, Casarotto S, Fecchio M, Rosanova M, Mariotti M, Lo Russo G, Palva JM, Nobili L, Massimini M (2015) Bistability breaks-off deterministic responses to intracortical stimulation during non-REM sleep. Neuroimage 112: 105-113. CrossRef Medline

Pompe B, Blidh P, Hoyer D, Eiselt M (1998) Using mutual information to measure coupling in the cardiorespiratory system. IEEE Eng Med Biol Mag 17:32-39. Medline

Puig MV, Ushimaru M, Kawaguchi Y (2008) Two distinct activity patterns of fast-spiking interneurons during neocortical UP states. Proc Natl Acad Sci U S A 105:8428-8433. CrossRef Medline

Quian Quiroga R, Panzeri S (2009) Extracting information from neuronal populations: information theory and decoding approaches. Nat Rev Neurosci 10:173-185. CrossRef Medline

Reinagel P, Reid RC (2000) Temporal coding of visual information in the thalamus. J Neurosci 20:5392-5400. Medline

Sachidhanandam S, Sreenivasan V, Kyriakatos A, Kremer Y, Petersen CC (2013) Membrane potential correlates of sensory perception in mouse barrel cortex. Nat Neurosci 16:1671-1677. CrossRef Medline

Saleem AB, Chadderton P, Apergis-Schoute J, Harris KD, Schultz SR (2010) Methods for predicting cortical UP and DOWN states from the phase of deep layer local field potentials. J Comput Neurosci 29:49-62. CrossRef Medline

Schreiber T (2000) Measuring information transfer. Phys Rev Lett 85:461464. CrossRef Medline

Shu Y, Hasenstaub A, McCormick DA (2003) Turning on and off recurrent balanced cortical activity. Nature 423:288-293. CrossRef Medline

Steriade M, Timofeev I, Grenier F (2001) Natural waking and sleep states: a view from inside neocortical neurons. J Neurophysiol 85:1969-1985. Medline

Steuer R, Kurths J, Daub CO, Weise J, Selbig J (2002) The mutual information: detecting and evaluating dependencies between variables. Bioinformatics 18:S231-S240. CrossRef Medline

Tauste Campo A, Martinez-Garcia M, Nácher V, Luna R, Romo R, Deco G (2015) Task-driven intra- and inter-area communications in primate cerebral cortex. Proc Natl Acad Sci U S A 112:4761-4766. CrossRef Medline

Vinck M, Batista-Brito R, Knoblich U, Cardin JA (2015a) Arousal and locomotion make distinct contributions to cortical activity patterns and visual encoding. Neuron 86:740-754. CrossRef Medline

Vinck M, Bos JJ, Van Mourik-Donga LA, Oplaat KT, Klein GA, Jackson JC, Gentet LJ, Pennartz CM (2015b) Cell-type and state-dependent synchronization among rodent somatosensory, visual, perirhinal cortex, and hippocampus CA1. Front Syst Neurosci 9:187. CrossRef Medline

Vyazovskiy VV, Olcese U, Lazimy YM, Faraguna U, Esser SK, Williams JC, Cirelli C, Tononi G (2009) Cortical firing and sleep homeostasis. Neuron 63:865-878. CrossRef Medline

Vyazovskiy VV, Olcese U, Hanlon EC, Nir Y, Cirelli C, Tononi G (2011) Local sleep in awake rats. Nature 472:443-447. CrossRef Medline

Wallisch P, Lusignan ME, Benayoun MD, Baker TI, Dickey AS, Hatsopoulos NG (2008) MATLAB for neuroscientists: an introduction to scientific computing in MATLAB, Ed 1. Boston: Academic.

Wang Q, Chen F, Xu W, Yang MH (2012) Object tracking via partial least squares analysis. IEEE Trans Image Process 21:4454-4465. CrossRef Medline

Wierenga CJ, Müllner FE, Rinke I, Keck T, Stein V, Bonhoeffer T (2010) Molecular and electrophysiological characterization of GFP-expressing CA1 interneurons in GAD65-GFP mice. PLoS One 5:e15915. CrossRef Medline

Witter MP, Naber PA, van Haeften T, Machielsen WC, Rombouts SA, Barkhof F, Scheltens P, Lopes da Silva FH (2000) Cortico-hippocampal communication by way of parallel parahippocampal-subicular pathways. Hippocampus 10:398-410. Medline

Ylinen A, Bragin A, Nádasdy Z, Jandó G, Szabó I, Sik A, Buzsáki G (1995) Sharp wave-associated high-frequency oscillation $(200 \mathrm{~Hz})$ in the intact hippocampus: network and intracellular mechanisms. J Neurosci 15: 30-46. Medline

Zhang S, Xu M, Kamigaki T, Hoang Do JP, Chang WC, Jenvay S, Miyamichi K, Luo L, Dan Y (2014) Selective attention: long-range and local circuits for top-down modulation of visual cortex processing. Science 345: 660-665. CrossRef Medline 